\title{
State-Space Geometry, Statistical Fluctuations, and Black Holes in String Theory
}

\author{
Stefano Bellucci and Bhupendra Nath Tiwari \\ INFN-Laboratori Nazionali di Frascati, Via E. Fermi 40, 00044 Frascati, Italy \\ Correspondence should be addressed to Stefano Bellucci; bellucci@lnf.infn.it \\ Received 5 February 2014; Accepted 8 April 2014; Published 7 May 2014 \\ Academic Editor: Christian Corda
}

Copyright ( 2014 S. Bellucci and B. N. Tiwari. This is an open access article distributed under the Creative Commons Attribution License, which permits unrestricted use, distribution, and reproduction in any medium, provided the original work is properly cited. The publication of this article was funded by $\mathrm{SCOAP}^{3}$.

\begin{abstract}
We study the state-space geometry of various extremal and nonextremal black holes in string theory. From the notion of the intrinsic geometry, we offer a state-space perspective to the black hole vacuum fluctuations. For a given black hole entropy, we explicate the intrinsic geometric meaning of the statistical fluctuations, local and global stability conditions, and long range statistical correlations. We provide a set of physical motivations pertaining to the extremal and nonextremal black holes, namely, the meaning of the chemical geometry and physics of correlation. We illustrate the state-space configurations for general charge extremal black holes. In sequel, we extend our analysis for various possible charge and anticharge nonextremal black holes. From the perspective of statistical fluctuation theory, we offer general remarks, future directions, and open issues towards the intrinsic geometric understanding of the vacuum fluctuations and black holes in string theory.
\end{abstract}

\section{Introduction}

In this paper, we study statistical properties of the charged and anticharged black hole configurations in string theory. Specifically, we illustrate that the components of the vacuum fluctuations define a set of local pair correlations against the parameters, for example, charges, anticharges, mass, and angular momenta. Our consideration follows from the notion of the thermodynamic geometry, mainly introduced by Weinhold [1,2] and Ruppeiner [3-9]. Importantly, this framework provides a simple platform to geometrically understand the statistical nature of local pair correlations and underlying structures pertaining to the vacuum phase transitions. In diverse contexts, the state-space geometric perspective offers an understanding of the phase structures of mixtures of gases, black hole configurations [10-26], generalized uncertainty principle [27], strong interactions, for example, hot QCD [28], quarkonium configurations [29], and some other systems, as well.

The main purpose of the present paper is to consider the state-space properties of various possible extremal and nonextremal black holes in string theory, in general. String theory [30], as the most promising framework to understand all possible fundamental interactions, celebrates the physics of black holes, in both the zero and the nonzero temperature domains. Our consideration hereby plays a crucial role in understanding the possible phases and stability of the string theory vacua. A further motivation follows from the consideration of the string theory black holes; namely, $\mathcal{N}=2$ supergravity arises as a low energy limit of the Type II string theory solution, admitting extremal black holes with the zero Hawking temperature and a nonzero macroscopic attractor entropy.

A priori, the entropy depends on a large number of scalar moduli arising from the compactification of the 10dimensional theory down to the 4-dimensional physical spacetime. This involves a 6-dimensional compactifying manifold. Interesting string theory compactifications involve $T^{6}, K_{3} \times T^{2}$, and Calabi-Yau manifolds. The macroscopic entropy exhibits a fixed point behavior under the radial flow of the scalar fields. In such cases, the near horizon geometry of an extremal black hole turns out to be an 
$A d S_{2} \times S^{2}$ manifold which describes the Bertotti-Robinson vacuum associated with the black hole. The area of the black hole horizon is $A$ and thus the macroscopic entropy [3142] is given as $S_{\text {macro }}=\pi\left|Z_{\infty}\right|^{2}$. This is known as the Ferrara-Kallosh-Strominger attractor mechanism, which, as the macroscopic consideration, requires a validity from the microscopic or statistical basis of the entropy. In this concern, there have been various investigations on the physics of black holes, for example, horizon properties [43, 44], counting of black hole microstates [45-47], spectrum of half-BPS states in $\mathcal{N}=4$ supersymmetric string theory [48], and fractionation of branes [49]. From the perspective of the fluctuation theory, our analysis is intended to provide the nature of the statistical structures of the extremal and nonextremal black hole configurations. The attractor configurations exist for the extremal black holes, in general. However, the corresponding nonextremal configurations exist in the throat approximation. In this direction, it is worth mentioning that there exists an extension of Sen entropy function formalism for $D_{1} D_{5}$ and $D_{2} D_{6} N S_{5}$ nonextremal configurations [5052]. In the throat approximation, these solutions, respectively, correspond to Schwarzschild black holes in $\mathrm{AdS}_{3} \times S^{3} \times T^{4}$ and $\mathrm{AdS}_{3} \times S^{2} \times S^{1} \times T^{4}$. In relation with the intrinsic statespace geometry, we will explore the statistical understanding of the attractor mechanism and the moduli space geometry and explain the vacuum fluctuations of the black brane configurations.

In this paper, we consider the state-space geometry of the spherical horizon topology black holes in four spacetime dimensions. These configurations carry a set of electric magnetic charges $\left(q_{i}, p_{i}\right)$. Due to the consideration of Strominger and Vafa [53], these charges are associated with an ensemble of weakly interacting $D$-branes. Following [53-59], it turns out that the charges $\left(q_{i}, p_{i}\right)$ are proportional to the number of electric and magnetic branes, which constitute the underlying ensemble of the chosen black hole. In the large charge limit, namely, when the number of such branes becomes large, we have treated the logarithm of the degeneracy of states of the statistical configuration as the Bekenstein-Hawking entropy of the associated string theory black holes. For the extremal black holes, the entropy is described in terms of the number of the constituent $D$-branes. For example, the two charge extremal configurations can be examined in terms of the winding modes and the momentum modes of an excited string carrying $n_{1}$ winding modes and $n_{p}$ momentum modes. Correspondingly, the state-space geometry of the nonextremal black holes is described by adding energy to the extremal $D$-branes configurations. This renders as the contribution of the clockwise and anticlockwise momenta in the Kaluza-Klein scenarios and that of the antibrane charges in general to the black hole entropy.

From the perspective of black hole thermodynamics, we describe the structure of the state-space geometry of fourdimensional extremal and nonextremal black holes in a given duality frame. Thus, when we take arbitrary variations over the charges $\left(q_{i}, p_{i}\right)$ on the electric and magnetic branes, the underlying statistical fluctuations are described by only the numbers of the constituent electric and magnetic branes.
From the perspective of the intrinsic state-space geometry, if one pretends that the notion of statistical fluctuations applies to intermediate regimes of the moduli space, then the attractor horizon configurations require an embedding to the higher dimensional intrinsic Riemanian manifold. Physically, such a higher dimensional manifold can be viewed as a possible blow-up of the attractor fixed point phasespace to a nontrivial moduli space. From the perspective of thermodynamic Ruppenier geometry, we have offered future directions and open issues in the conclusion. We leave the explicit consideration of these matters open for further research.

In Section 2, we define the general notion of vacuum fluctuations. This offers the physical meaning of the statespace geometry. In Section 3, we provide a brief review of statistical fluctuations. In particular, for a given black hole entropy, we firstly explicate the statistical meaning of statespace surface and then offer the general meaning of the local and global stability conditions and long range statistical correlations. In Section 4, we provide a set of physical motivations pertaining to the extremal and nonextremal black holes, the meaning of Wienhold chemical geometry, and the physics of correlation. In Section 5, we consider state-space configurations pertaining to the extremal black holes and explicate our analysis for the two and three charge configurations. In Section 6, we extend the above analysis for the four, six, and eight charge-anticharge nonextremal black holes. Finally, Section 7 provides general remarks, conclusion and outlook, and future directions and open issues towards the application of string theory.

\section{Definition of State-Space Geometry}

Considering the fact that the black hole configurations in string theory introduce the notion of vacuum, it turns out for any thermodynamic system, that there exist equilibrium thermodynamic states given by the maxima of the entropy. These states may be represented by points on the state-space. Along with the laws of the equilibrium thermodynamics, the theory of fluctuations leads to the intrinsic Riemannian geometric structure on the space of equilibrium states $[8,9]$. The invariant distance between two arbitrary equilibrium states is inversely proportional to the fluctuations connecting the two states. In particular, a less probable fluctuation means that the states are far apart. For a given set of states $\left\{X_{i}\right\}$, the state-space metric tensor is defined by

$$
g_{i j}(X)=-\partial_{i} \partial_{j} S\left(X_{1}, X_{2}, \ldots, X_{n}\right) .
$$

A physical motivation of (1) can be given as follows. Up to the second order approximation, the Taylor expansion of the entropy $S\left(X_{1}, X_{2}, \ldots, X_{n}\right)$ yields

$$
S-S_{0}=-\frac{1}{2} \sum_{i=1}^{n} g_{i j} \Delta X^{i} \Delta X^{j},
$$

where

$$
g_{i j}:=-\frac{\partial^{2} S\left(X_{1}, X_{2}, \ldots, X_{n}\right)}{\partial X^{i} \partial X^{j}}=g_{j i}
$$


is called the state-space metric tensor. In the present investigation, we consider the state-space variables $\left\{X_{1}, X_{2}, \ldots, X_{n}\right\}$ as the parameters of the ensemble of the microstates of the underlying microscopic configuration (e.g., conformal field theory [60], black hole conformal field theory [61], and hidden conformal field theory $[62,63])$, which defines the corresponding macroscopic thermodynamic configuration. Physically, the state-space geometry can be understood as the intrinsic Riemannian geometry involving the parameters of the underlying microscopic statistical theory. In practice, we will consider the variables $\left\{X_{1}, X_{2}, \ldots, X_{n}\right\}$ as the parameters, namely, charges, anticharges, and others if any, of the corresponding low energy limit of the string theory, for example, $\mathcal{N}=2$ supergravity. In the limit, when all the variables, namely, $\left\{X_{1}, X_{2}, \ldots, X_{n}\right\}$, are thermodynamic, the state-space metric tensor equation (1) reduces to the corresponding Ruppenier metric tensor. In the discrete limit, the relative coordinates $\Delta X^{i}$ are defined as $\Delta X^{i}:=X^{i}-X_{0}^{i}$, for given $\left\{X_{0}^{i}\right\} \in M_{n}$. In the Gaussian approximation, the probability distribution has the following form:

$$
P\left(X_{1}, X_{2}, \ldots, X_{n}\right)=A \exp \left(-\frac{1}{2} g_{i j} \Delta X^{i} \Delta X^{j}\right) .
$$

With the normalization

$$
\int \prod_{i} d X_{i} P\left(X_{1}, X_{2}, \ldots, X_{n}\right)=1,
$$

we have the following probability distribution:

$$
P\left(X_{1}, X_{2}, \ldots, X_{n}\right)=\frac{\sqrt{g(X)}}{(2 \pi)^{n / 2}} \exp \left(-\frac{1}{2} g_{i j} d X^{i} \otimes d X^{j}\right),
$$

where $g_{i j}$ now, in a strict mathematical sense, is properly defined as the inner product $g\left(\partial / \partial X^{i}, \partial / \partial X^{j}\right)$ on the corresponding tangent space $T\left(M_{n}\right) \times T\left(M_{n}\right)$. In this connotation, the determinant of the state-space metric tensor,

$$
g(X):=\left\|g_{i j}\right\|,
$$

can be understood as the determinant of the corresponding matrix $\left[g_{i j}\right]_{n \times n}$. For a given state-space manifold $\left(M_{n}, g\right)$, we will think of $\left\{d X^{i}\right\}$ as the basis of the cotangent space $T^{\star}\left(M_{n}\right)$. In the subsequent analysis, by taking an account of the fact that the physical vacuum is neutral, we will choose $X_{0}^{i}=0$.

\section{Statistical Fluctuations}

3.1. Black Hole Entropy. As a first exercise, we have illustrated thermodynamic state-space geometry for the two charge extremal black holes with electric charge $q$ and magnetic charge $p$. The next step has thence been to examine the thermodynamic geometry at an attractor fixed point(s) for the extremal black holes as the maxima of their macroscopic entropy $S(q, p)$. Later on, the state-space geometry of nonextremal counterparts has as well been analyzed. In this investigation, we demonstrate that the state-space correlations of nonextremal black holes modulate relatively more swiftly to an equilibrium statistical basis than those of the corresponding extremal solutions.
3.2. State-Space Surface. The Ruppenier metric on the statespace $\left(M_{2}, g\right)$ of two charge black holes is defined by

$$
\begin{gathered}
g_{q q}=-\frac{\partial^{2} S(q, p)}{\partial q^{2}}, \quad g_{q p}=-\frac{\partial^{2} S(q, p)}{\partial q \partial p}, \\
g_{p p}=-\frac{\partial^{2} S(q, p)}{\partial p^{2}} .
\end{gathered}
$$

Subsequently, the components of the state-space metric tensor are associated with the respective statistical pair correlation functions. It is worth mentioning that the coordinates on the state-space manifold are the parameters of the microscopic boundary conformal field theory which is dual the black hole space-time solution. This is because the underlying state-space metric tensor comprises of the Gaussian fluctuations of the entropy which is the function of the number of the branes and antibranes. For the chosen black hole configuration, the local stability of the underlying statistical system requires both principle minors to be positive. In this setup, the diagonal components of the statespace metric tensor, namely, $\left\{g_{x_{i} x_{i}} \mid x_{i}=(n, m)\right\}$, signify the heat capacities of the system. This requires that the diagonal components of the state-space metric tensor

$$
g_{x_{i} x_{i}}>0, \quad i=n, m
$$

be positive definite. In this investigation, we discuss the significance of the above observation for the eight parameter nonextremal black brane configurations in string theory. From the notion of the relative scaling property, we will demonstrate the nature of the brane-brane pair correlations; namely, from the perspective of the intrinsic Riemannian geometry, the stability properties of the eight parameter black branes are examined from the positivity of the principle minors of the space-state metric tensor. For the Gaussian fluctuations of the two charge equilibrium statistical configurations, the existence of a positive definite volume form on the state-space manifold $\left(M_{2}(R), g\right)$ imposes such a global stability condition. In particular, the above configuration leads to a stable statistical basis if the determinant of the statespace metric tensor,

$$
\|g\|=S_{n n} S_{m m}-S_{n m}^{2}
$$

remains positive. Indeed, for the two charge black brane configurations, the geometric quantities corresponding to the underlying state-space manifold elucidate typical features of the Gaussian fluctuations about an ensemble of equilibrium brane microstates. In this case, we see that the Christoffel connections on the $\left(M_{2}, g\right)$ are defined by

$$
\Gamma_{i j k}=g_{i j, k}+g_{i k, j}-g_{j k, i} .
$$

The only nonzero Riemann curvature tensor is

$$
R_{q p q p}=\frac{N}{D}
$$


where

$$
\begin{aligned}
N:= & S_{p p} S_{q q q} S_{q p p}+S_{q p} S_{q q p} S_{q p p} \\
& +S_{q q} S_{q q p} S_{p p p}-S_{q p} S_{q q q} S_{p p p} \\
& -S_{q q} S_{q p p}^{2}-S_{p p} S_{q q p}^{2}, \\
& D:=\left(S_{q q} S_{p p}-S_{q p}^{2}\right)^{2} .
\end{aligned}
$$

The scalar curvature and the corresponding $R_{i j k l}$ of an arbitrary two-dimensional intrinsic state-space manifold $\left(M_{2}(R), g\right)$ may be given as

$$
R(q, p)=\frac{2}{\|g\|} R_{q p q p}(q, p) .
$$

3.3. Stability Conditions. For a given set of variables $\left\{X^{1}, X^{2}, \ldots, X^{n}\right\}$, the local stability of the underlying statespace configuration demands the positivity of the heat capacities:

$$
\left\{g_{i i}\left(X^{i}\right)>0 ; \forall i=1,2, \ldots, n\right\} .
$$

Physically, the principle components of the state-space metric tensor $\left\{g_{i i}\left(X^{i}\right) \mid i=1,2, \ldots, n\right\}$ signify a set of definite heat capacities (or the related compressibilities), whose positivity apprises that the black hole solution complies an underlying, locally in equilibrium, statistical configuration. Notice further that the positivity of principle components is not sufficient to insure the global stability of the chosen configuration and thus one may only achieve a locally stable equilibriumstatistical configuration. In fact, the global stability condition constraint over the allowed domain of the parameters of black hole configurations requires that all the principle components and all the principle minors of the metric tensor must be strictly positive definite [6]. The above stability conditions require that the following set of equations must be simultaneously satisfied:

$$
\begin{aligned}
p_{0} & :=1, \\
p_{1}: & =g_{11}>0, \\
p_{2}: & =\left|\begin{array}{ll}
g_{11} & g_{12} \\
g_{12} & g_{22}
\end{array}\right|>0, \\
p_{3}:= & \left|\begin{array}{lll}
g_{11} & g_{12} & g_{13} \\
g_{12} & g_{22} & g_{23} \\
g_{13} & g_{23} & g_{33}
\end{array}\right|>0, \\
& \vdots \\
p_{n}: & =\|g\|>0 .
\end{aligned}
$$

3.4. Long Range Correlations. The thermodynamic scalar curvature of the state-space manifold is proportional to the correlation volume [6]. Physically, the scalar curvature signifies the interaction(s) of the underlying statistical system.
Ruppenier has in particular noticed for the black holes in general relativity that the scalar curvature

$$
R(X) \sim \xi^{d},
$$

where $d$ is the spatial dimension of the statistical system and the $\xi$ fixes the physical scale [6]. The limit $R(X) \rightarrow$ $\infty$ indicates the existence of certain critical points or phase transitions in the underlying statistical system. The fact that "all the statistical degrees of freedom of a black hole live on the black hole event horizon" signifies that the statespace scalar curvature, as the intrinsic geometric invariant, indicates an average number of correlated Plank areas on the event horizon of the black hole [8]. In this concern, [9] offers interesting physical properties of the thermodynamic scalar curvature and phase transitions in Kerr-Newman black holes. Ruppeiner [6] has further conjectured that the global correlations can be expressed by the following arguments: (a) the zero state-space scalar curvature indicates certain bits of information on the event horizon, fluctuating independently of each other; (b) the diverging scalar curvature signals a phase transition indicating highly correlated pixels of the information.

\section{Some Physical Motivations}

4.1. Extremal Black Holes. The state-space of the extremal black hole configuration is a reduced space comprising of the states which respect the extremality (BPS) condition. The state-spaces of the extremal black holes show an intrinsic geometric description. Our intrinsic geometric analysis offers a possible zero temperature characterization of the limiting extremal black brane attractors. From the gauge/gravity correspondence, the existence of state-space geometry could be relevant to the boundary gauge theories, which have finitely many countable sets of conformal field theory states.

4.2. Nonextremal Black Holes. We will analyze the statespace geometry of nonextremal black holes by the addition of antibrane charge(s) to the entropy of the corresponding extremal black holes. To interrogate the stability of a chosen black hole system, we will investigate the question that the underlying metric $g_{i j}\left(X_{i}\right)=-\partial_{i} \partial_{j} S\left(X_{1}, X_{2}, \ldots, X_{n}\right)$ should provide a nondegenerate state-space manifold. The exact dependence varies case to case. In the next section, we will proceed in our analysis with an increasing number of the brane charges and antibrane charges.

4.3. Chemical Geometry. The thermodynamic configurations of nonextremal black holes in string theory with small statistical fluctuations in a "canonical" ensemble are stable if the following inequality holds:

$$
\left\|\partial_{i} \partial_{j} S\left(X_{1}, X_{2}, \ldots, X_{n}\right)\right\|<0 .
$$

The thermal fluctuations of nonextremal black holes, when considered in the canonical ensemble, give a closer approximation to the microcanonical entropy:

$$
S=S_{0}-\frac{1}{2} \ln \left(C T^{2}\right)+\cdots .
$$


In (20), the $S_{0}$ is the entropy in the "canonical" ensemble and $C$ is the specific heat of the black hole statistical configuration. At low temperature, the quantum effects dominate and the above expansion does not hold anymore. The stability condition of the canonical ensemble is just $C>0$. In other words, the Hessian function of the internal energy with respect to the chemical variables, namely, $\left\{x_{1}, x_{2}, \ldots, x_{n}\right\}$, remains positive definite. Hence, the energy as the function of the $\left\{x_{1}, x_{2}, \ldots, x_{n}\right\}$ satisfies the following condition:

$$
\left\|\partial_{i} \partial_{j} E\left(x_{1}, x_{2}, \ldots, x_{n}\right)\right\|>0 .
$$

The state-space coordinates $\left\{X^{i}\right\}$ and intensive chemical variables $\left\{x_{i}\right\}$ are conjugate to each other. In particular, the $\left\{X^{i}\right\}$ are defined as the Legendre transform of $\left\{x_{i}\right\}$, and thus we have

$$
X^{i}:=\frac{\partial S(x)}{\partial x_{i}}
$$

4.4. Physics of Correlation. Geometrically, the positivity of the heat capacity $C>0$ turns out to be the positivity condition of $g_{i j}>0$, for a given $i$. In many cases, the state-space stability restriction on the parameters of the black hole corresponds to the situation away from the extremality condition; namely, $r_{+}=r_{-}$. Far from the extremality condition, even at the zero antibrane charge or angular momentum, we find that there is a finite value of the thermodynamic scalar curvature, unlike the nonrotating or only brane-charged configurations. It turns out that the state-space geometry of the two charge extremal configurations is flat. Thus, the Einstein-Hilbert contributions lead to a noninteracting statistical system. At the tree level, some black hole configurations turn out to be ill-defined, as well. However, we anticipate that the corresponding state-space configuration would become welldefined when a sufficient number of higher derivative corrections [64-67] are taken into account with respect to the $\alpha^{\prime}$-corrections and the string loop $l_{s}$ corrections. For the BTZ black holes [13], we notice that the large entropy limit turns out to be the stability bound, beyond which the underlying quantum effects dominate.

For the black hole in string theory, the Ricci scalar of the state-space geometry is anticipated to be positive definite with finitely many higher order corrections. For nonextremal black brane configurations, which are far from the extremality condition, such effects have been seen from the nature of the state-space scalar curvature $R\left(S\left(X_{1}, X_{2}, \ldots, X_{n}\right)\right.$ ). Indeed, $[12,14]$ indicate that the limiting state-space scalar curvature $\left.R\left(S\left(X_{1}, X_{2}, \ldots, X_{n}\right)\right)\right|_{\text {no anticharge }} \neq 0$ gives a set of stability bounds on the statistical parameters. Thus, our consideration yields a classification of the domain of the parameters and global correlation of a nonextremal black hole.

4.5. String Theory Perspective. In this subsection, we recall a brief notion of entropy of a general string theory black brane configuration from the viewpoint of the counting of the black hole microstates $[53,53-59,68]$. Given a string theory configuration, the choice of compactification [30] chosen is the factorization of the type $\mathscr{M}_{(3,1)} \times M_{6}$, where $M_{6}$ is a compact internal manifold. From the perspective of statistical ensemble theory, we will express the entropy of a nonextremal black hole as the function of the numbers of branes and antibranes. Namely, for the charged black holes, the electric and magnetic charges $\left(q_{i}, p_{i}\right)$ form a coordinate chart on the state-space manifold. In this case, for a given ensemble of $D$-branes, the coordinate $q_{i}$ is defined as the number of the electric branes and $p_{i}$ as the number of the magnetic branes. Towards the end of this paper, we will offer further motivation for the consideration of the statespace geometry of large charged nonspherical horizon black holes in spacetime dimensions $D \geq 5$. In this concern, [68] plays a central role towards the formation of the lower dimensional black hole configuration. Namely, for the torus compatifications, the exotic branes play an important role concerning the physical properties of supertubes, the $D_{0}-F_{1}$ system and associated counting of the black hole microstates.

In what follows, we consider the four-dimensional string theory black holes in a given duality basis of the charges $\left(q_{i}, p_{i}\right)$. From the perspective of string theory, the exotic branes and nongeometric configurations offer interesting fronts for the black holes in three spacetime dimensions. In general, such configurations could carry a dipole or a higher pole charge, and they leave the four-dimension black hole configuration asymptotically flat. In fact, for the spacetime dimensions $D \geq 4$, [68] shows that a charge particle corresponds to an underlying gauge field, modulo $U$-duality transformations. From the perspective of nonextremal black holes, by taking appropriate boundary condition, namely, the unit asymptotic limit of the harmonic function which defines the spacetime metric, one can choose the spacetime regions such that the supertube effects arising from nonexotic branes can effectively be put off in an asymptotically flat space [68]. This allows one to compute the Arnowitt-DeserMisner (ADM) mass of the asymptotic black hole. From the viewpoint of the statistical investigation, the dependence of the mass to the entropy of a nonextremal black hole comes from the contribution of the antibranes to the counting degeneracy of the states.

\section{Extremal Black Holes in String Theory}

5.1. Two Charge Configurations. The state-space geometry of the two charge extremal configurations is analyzed in terms of the winding modes and the momentum modes of an excited string carrying $n_{1}$ winding modes and $n_{p}$ momentum modes. In the large charge limit, the microscopic entropy obtained by the degeneracy of the underlying conformal field theory states reduces to the following expression:

$$
S_{\text {micro }}=2 \sqrt{2 n_{1} n_{p}} .
$$

The microscopic counting can be accomplished by considering an ensemble of weakly interacting $D$-branes [54]. The counting entropy and the macroscopic attractor entropy of the two charge black holes in string theory which have a $n_{4}$ 
number of $D_{4}$ branes and a $n_{0}$ number of $D_{0}$ branes match and thus we have

$$
S_{\text {micro }}=2 \pi \sqrt{n_{0} n_{4}}=S_{\text {macro }} .
$$

In this case, the components of underlying state-space metric tensor are

$$
\begin{gathered}
g_{n_{0} n_{0}}=\frac{\pi}{2 n_{0}} \sqrt{\frac{n_{4}}{n_{0}}}, \quad g_{n_{0} n_{4}}=-\frac{\pi}{2} \frac{1}{\sqrt{n_{0} n_{4}}}, \\
g_{n_{4} n_{4}}=\frac{\pi}{2 n_{4}} \sqrt{\frac{n_{0}}{n_{4}}} .
\end{gathered}
$$

The diagonal pair correlation functions remain positive definite:

$$
g_{n_{i} n_{i}}>0 \quad \forall i \in\{0,4\} \mid n_{i}>0, \quad g_{n_{4} n_{4}}>0, \quad \forall\left(n_{0}, n_{4}\right) .
$$

For distinct $i, j \in\{0,4\}$, the state-space pair correlation functions admit

$$
\frac{g_{i i}}{g_{j j}}=\left(\frac{n_{j}}{n_{i}}\right)^{2}, \quad \frac{g_{i j}}{g_{i i}}=-\frac{n_{i}}{n_{j}} .
$$

The global properties of fluctuating two charge $D_{0}-D_{4}$ extremal configurations are determined by possible principle minors. The first minor constraint $p_{1}>0$ directly follows from the positivity of the first component of metric tensor:

$$
p_{1}=\frac{\pi}{2 n_{0}} \sqrt{\frac{n_{4}}{n_{0}}} .
$$

The determinant of the metric tensor $p_{2}:=g\left(n_{0}, n_{4}\right)$ vanishes identically for all allowed values of the parameters. Thus, the leading order large charge extremal black branes having (i) a $n_{0}$ number of $D_{0}$-branes and a $n_{4}$ number of $D_{4}$ or (ii) excited strings with a $n_{1}$ number of windings and a $n_{p}$ number of momenta, where either set of charges forms local coordinates on the state-space manifold, find degenerate intrinsic state-space configurations. For a given configuration entropy $S_{0}:=2 \pi c$, the constant entropy curve can be depicted as the rectangular hyperbola

$$
n_{0} n_{4}=c^{2}
$$

The intrinsic state-space configuration depends on the attractor values of the scalar fields which arise from the chosen string compactification. Thus, the possible statespace Ruppenier geometry may become well-defined against further higher derivative $\alpha^{\prime}$-corrections. In particular, the determinant of the state-space metric tensor may take positive/negative definite values over the domain of brane charges. We will illustrate this point in a bit more detail in the subsequent consideration with a higher number of charges and anticharges.
5.2. Three Charge Configurations. From the consideration of the two derivative Einstein-Hilbert action, [53] shows that the leading order entropy of the three charge $D_{1}-D_{5}-P$ extremal black holes is

$$
S_{\text {micro }}=2 \pi \sqrt{n_{1} n_{5} n_{p}}=S_{\text {macro }} .
$$

The concerned components of state-space metric tensor are given in Appendix A. Hereby, it follows further that the local state-space metric constraints are satisfied as

$$
g_{n_{i} n_{i}}>0 \quad \forall i \in\{1,5, p\} \mid n_{i}>0 .
$$

For distinct $i, j \in\{1,5\}$ and $p$, the list of relative correlation functions is depicted in Appendix A. Further, we see that the local stabilities pertaining to the lines and two-dimensional surfaces of the state-space manifold are measured as

$$
p_{1}=\frac{\pi}{2 n_{1}} \sqrt{\frac{n_{5} n_{p}}{n_{1}}}, \quad p_{2}=-\frac{\pi^{2}}{4 n_{1} n_{5}^{2} n_{p}}\left(n_{p}^{2} n_{1}+n_{5}^{3}\right) .
$$

The stability of the entire equilibrium phase-space configurations of the $D_{1}-D_{5}-P$ extremal black holes is determined by the $p_{3}:=g$ determinant of the state-space metric tensor:

$$
\|g\|=-\frac{1}{2} \pi^{3}\left(n_{1} n_{5} n_{p}\right)^{-1 / 2} .
$$

The universal nature of statistical interactions and the other properties concerning Maldacena, Strominger, and Witten (MSW) rotating black branes [55] are elucidated by the statespace scalar curvature:

$$
R\left(n_{1}, n_{5}, n_{p}\right)=\frac{3}{4 \pi \sqrt{n_{1} n_{5} n_{p}}} .
$$

The constant entropy (or scalar curvature) curve defining the state-space manifold is the higher dimensional hyperbola:

$$
n_{1} n_{5} n_{p}=c^{2}
$$

where $c$ takes respective values of $\left(c_{S}, c_{R}\right)=\left(S_{0} / 2 \pi, 3 / 4 \pi R_{0}\right)$. In $[12,14,17,18]$, we have shown that similar results hold for the state-space configuration of the four charge extremal black holes.

\section{Nonextremal Black Holes in String Theory}

6.1. Four Charge Configurations. The state-space configuration of the nonextremal $D_{1}-D_{5}$ black holes is considered with nonzero momenta along the clockwise and anticlockwise directions of the Kaluza-Klein compactification circle $S^{1}$. Following [56], the microscopic entropy and the macroscopic entropy match for given total mass and brane charges :

$$
S_{\text {micro }}=2 \pi \sqrt{n_{1} n_{5}}\left(\sqrt{n_{p}}+\sqrt{\overline{n_{p}}}\right)=S_{\text {macro }} .
$$

The state-space covariant metric tensor is defined as a negative Hessian matrix of the entropy with respect to the number of $D_{1}, D_{5}$ branes $\left\{n_{i} \mid i=1,5\right\}$ and clockwise-anticlockwise 
Kaluza-Klein momentum charges $\left\{n_{p}, \overline{n_{p}}\right\}$. Herewith, we find that the components of the metric tensor take elegant forms. The corresponding expressions are given in Appendix B. As in the case of the extremal configurations, the state-space metric satisfies the following constraints:

$$
g_{n_{i} n_{i}}>0, \quad \forall i=1,5 ; \quad g_{n_{a} n_{a}}>0, \quad \forall a=p, \bar{p}
$$

Furthermore, the scaling relations for distinct $i, j \in$ $\{1,5\}$ and $p$, concerning the list of relative correlation functions, are offered in Appendix B. In this case, we find that the stability criteria of the possible surfaces and hypersurfaces of the underlying state-space configuration are determined by the positivity of the following principle minors:

$$
\begin{aligned}
& p_{0}=1, \quad p_{1}=\frac{\pi}{2} \sqrt{\frac{n_{5}}{n_{1}^{3}}}\left(\sqrt{n_{p}}+\sqrt{\overline{n_{p}}}\right), \\
& p_{2}=0, \quad p_{3}=-\frac{1}{2 n_{p}} \frac{\pi^{3}}{\sqrt{n_{1} n_{5}}}\left(\sqrt{n_{p}}+\sqrt{\overline{n_{p}}}\right) .
\end{aligned}
$$

The complete local stability of the full nonextremal $D_{1}-D_{5}$ black brane state-space configuration is ascertained by the positivity of the determinant of the metric tensor:

$$
g\left(n_{1}, n_{5}, n_{p}, \overline{n_{p}}\right)=-\frac{1}{4} \frac{\pi^{4}}{\left(n_{p} \overline{n_{p}}\right)^{3 / 2}}\left(\sqrt{n_{p}}+\sqrt{\overline{n_{p}}}\right)^{2}
$$

The global state-space properties concerning the four charge nonextremal $D_{1}-D_{5}$ black holes are determined by the regularity of the invariant scalar curvature:

$$
R\left(n_{1}, n_{5}, n_{p}, \overline{n_{p}}\right)=\frac{9}{4 \pi \sqrt{n_{1} n_{5}}}\left(\sqrt{n_{p}}+\sqrt{\overline{n_{p}}}\right)^{-6} f\left(n_{p}, \overline{n_{p}}\right)
$$

where the function $f\left(n_{p}, \overline{n_{p}}\right)$ of two momenta $\left(n_{p}, \overline{n_{p}}\right)$ running in opposite directions of the Kaluza-Klein circle $S^{1}$ has been defined as

$$
\begin{aligned}
f\left(n_{p}, \overline{n_{p}}\right):= & n_{p}^{5 / 2}+10 n_{p}^{3 / 2} \bar{n}_{p}+5 n_{p}^{1 / 2}{\overline{n_{p}}}^{2} \\
& +5 n_{p}^{2}{\overline{n_{p}}}^{1 / 2}+10 n_{p}{\overline{n_{p}}}^{3 / 2}+\bar{n}_{p}^{5 / 2}
\end{aligned}
$$

By noticing the Pascal coefficient structure in (41), we see that the above function $f\left(n_{p}, \overline{n_{p}}\right)$ can be factorized as

$$
f\left(n_{p}, \overline{n_{p}}\right)=\left(n_{p}+\overline{n_{p}}\right)^{5}
$$

Thus, (40) leads to the following state-space scalar curvature:

$$
R\left(n_{1}, n_{5}, n_{p}, \overline{n_{p}}\right)=\frac{9}{4 \pi \sqrt{n_{1} n_{5}}} \times\left(\frac{1}{\sqrt{n_{p}}+\sqrt{\overline{n_{p}}}}\right) .
$$

In the large charge limit, the nonextremal $D_{1}-D_{5}$ black branes have a nonvanishing small scalar curvature function on the state-space manifold $\left(M_{4}, g\right)$. This implies an almost everywhere weakly interacting statistical basis. In this case, the constant entropy hypersurface is defined by the curve

$$
\frac{c^{2}}{n_{1} n_{5}}=\left(\sqrt{n_{p}}+\sqrt{\overline{n_{p}}}\right)^{2} .
$$

As in the case of two charge $D_{0}-D_{4}$ extremal black holes and $D_{1}-D_{5}-P$ extremal black holes, the constant $c$ takes the same value of $c:=S_{0}^{2} / 4 \pi^{2}$. For a given state-space scalar curvature $k$, the constant state-space curvature curves take the following form:

$$
f\left(n_{p}, \overline{n_{p}}\right)=k \sqrt{n_{1} n_{5}}\left(\sqrt{n_{p}}+\sqrt{\overline{n_{p}}}\right)^{6} .
$$

6.2. Six Charge Configurations. We now extrapolate the statespace geometry of four charge nonextremal $D_{1}-D_{5}$ solutions for nonlarge charges, where we are no longer close to an ensemble of supersymmetric states. In [57], the computation of the entropy of all such special extremal and near-extremal black hole configurations has been considered. The leading order entropy as a function of charges $\left\{n_{i}\right\}$ and anticharges $\left\{m_{i}\right\}$ is

$$
\begin{aligned}
& S\left(n_{1}, m_{1}, n_{2}, m_{2}, n_{3}, m_{3}\right) \\
& \quad:=2 \pi\left(\sqrt{n_{1}}+\sqrt{m_{1}}\right)\left(\sqrt{n_{2}}+\sqrt{m_{2}}\right)\left(\sqrt{n_{3}}+\sqrt{m_{3}}\right) .
\end{aligned}
$$

For given charges $i, j \in A_{1}:=\left\{n_{1}, m_{1}\right\} ; k, l \in A_{2}:=\left\{n_{2}, m_{2}\right\}$; and $m, n \in A_{3}:=\left\{n_{3}, m_{3}\right\}$, the intrinsic state-space pair correlations are in precise accordance with the underlying macroscopic attractor configurations which are being disclosed in the special leading order limit of the nonextremal $D_{1}-D_{5}$ solutions. The components of the covariant statespace metric tensor over generic nonlarge charge domains are not difficult to compute, and, indeed, we have offered their corresponding expressions in Appendix C.

For all finite $\left(n_{i}, m_{i}\right), i=1,2,3$, the components involving brane-brane state-space correlations $g_{n_{i} n_{i}}$ and antibraneantibrane state-space correlations $g_{m_{i} m_{i}}$ satisfy the following positivity conditions:

$$
g_{n_{i} n_{i}}>0, \quad g_{m_{i} m_{i}}>0
$$

The distinct $\left\{n_{i}, m_{i} \mid i \in\{1,2,3\}\right\}$ describing six charge string theory black holes have three types of relative pair correlation functions. The corresponding expressions of the relative statistical correlation functions are given in Appendix C.

Notice hereby that the scaling relations remain similar to those obtained in the previous case, except that (i) the number of relative correlation functions has been increased, and (ii) the set of cross ratios, namely, $\left\{g_{i j} / g_{k l}, g_{k l} / g_{m n}, g_{i j} / g_{m n}\right\}$ being zero in the previous case, becomes ill-defined for the six charge state-space configurations. Inspecting the specific pair of distinct charge sets $A_{i}$ and $A_{j}$, there are now 24 types of nontrivial relative correlation functions. The set of principle 
components denominator ratios computed from the above state-space metric tensor reduces to

$$
\frac{g_{i j}}{g_{k k}}=0, \quad \forall i, j, k \in\left\{n_{1}, m_{1}, n_{2}, m_{2}, n_{3}, m_{3}\right\} .
$$

For given $i, j \in A_{1}:=\left\{n_{1}, m_{1}\right\} ; k, l \in A_{2}:=\left\{n_{2}, m_{2}\right\} ; m, n \in$ $A_{3}:=\left\{n_{3}, m_{3}\right\}$, and $g_{n_{i} m_{i}}=0$, there are the total 15 types of trivial relative correlation functions. There are five such trivial ratios in each family $\left\{A_{i} \mid i=1,2,3\right\}$. The local stability of the higher charged string theory nonextremal black holes is given by

$$
\begin{aligned}
p_{1}= & \frac{\pi}{2 n_{1}^{3 / 2}}\left(\sqrt{n_{2}}+\sqrt{m_{2}}\right)\left(\sqrt{n_{3}}+\sqrt{m_{3}}\right), \\
p_{2}= & \frac{1}{4} \frac{\pi^{2}}{\left(n_{1} m_{1}\right)^{3 / 2}}\left(\sqrt{n_{2}}+\sqrt{m_{2}}\right)^{2}\left(\sqrt{n_{3}}+\sqrt{m_{3}}\right)^{2}, \\
p_{3}= & \frac{1}{8} \frac{\pi^{3}}{\left(n_{1} m_{1} n_{2}\right)^{3 / 2}} \sqrt{m_{2}}\left(\sqrt{n_{3}}+\sqrt{m_{3}}\right)^{3} \\
& \times\left(\sqrt{n_{2}}+\sqrt{m_{2}}\right)\left(\sqrt{n_{1}}+\sqrt{m_{1}}\right), \\
p_{4}= & 0 .
\end{aligned}
$$

The principle minor $p_{5}$ remains nonvanishing for all values of charges on the constituent brane and antibranes. In general, by an explicit calculation, we find that the hyper-surface minor $p_{5}$ takes the following nontrivial value:

$$
\begin{aligned}
p_{5}= & -\frac{1}{8} \frac{\pi^{5}}{\left(n_{1} m_{1} n_{2} m_{2}\right)^{3 / 2} n_{3}}\left(\sqrt{n_{1}}+\sqrt{m_{1}}\right)^{3} \\
& \times\left(\sqrt{n_{2}}+\sqrt{m_{2}}\right)^{3}\left(\sqrt{n_{3}}+\sqrt{m_{3}}\right)^{3} .
\end{aligned}
$$

Specifically, for an identical value of the brane and antibrane charges, the minor $p_{5}$ reduces to

$$
p_{5}(k)=-64 \frac{\pi^{5}}{k^{5 / 2}} .
$$

The global stability on the full state-space configuration is carried forward by computing the determinant of the metric tensor:

$$
\begin{aligned}
\|g\|= & -\frac{1}{16} \frac{\pi^{6}}{\left(n_{1} m_{1} n_{2} m_{2} n_{3} m_{3}\right)^{3 / 2}}\left(\sqrt{n_{1}}+\sqrt{m_{1}}\right)^{4} \\
& \times\left(\sqrt{n_{2}}+\sqrt{m_{2}}\right)^{4}\left(\sqrt{n_{3}}+\sqrt{m_{3}}\right)^{4} .
\end{aligned}
$$

The underlying state-space configuration remains nondegenerate for the domain of given nonzero brane antibrane charges, except for extreme values of the brane and antibrane charges $\left\{n_{i}, m_{i}\right\}$, when they belong to the set

$$
\begin{aligned}
B:=\{ & \left(n_{1}, n_{2}, n_{3}, m_{1}, m_{2}, m_{3}\right) \mid \\
& \left.\left(n_{i}, m_{i}\right)=(0,0),(\infty, \infty), \text { some } i\right\},
\end{aligned}
$$

among the given brane-antibrane pairs $\left\{\left(n_{1}, m_{1}\right),\left(n_{2}\right.\right.$, $\left.\left.m_{2}\right),\left(n_{3}, m_{3}\right)\right\}$. The component $R_{n_{1} n_{2} m_{3} m_{4}}$ diverges at the roots of the two variables polynomials defined as the functions of brane and antibrane charges:

$$
\begin{aligned}
& f_{1}\left(n_{2}, m_{2}\right)=n_{2}^{4} m_{2}^{3}+2\left(n_{2} m_{2}\right)^{7 / 2}+n_{2}^{3} m_{2}^{4}, \\
& f_{2}\left(n_{3}, m_{3}\right)=m_{3}^{9 / 2} n_{3}^{4}+n_{3}^{4} m_{3}^{9 / 2} .
\end{aligned}
$$

However, the component $R_{n_{3}, m_{3}, n_{3}, m_{3}}$ with an equal number of brane and antibrane charges diverges at a root of a single higher degree polynomial:

$$
\begin{aligned}
f\left(n_{1}, m_{1}, n_{2}, m_{2}, n_{3}, m_{3}\right) & :=n_{2}^{4} m_{2}^{3} n_{3}^{9 / 2} m_{3}^{4}+n_{2}^{4} m_{2}^{3} n_{3}^{4} m_{3}^{9 / 2} \\
& +2 n_{2}^{7 / 2} m_{2}^{7 / 2} n_{3}^{9 / 2} m_{3}^{4}+2 n_{2}^{7 / 2} m_{2}^{7 / 2} n_{3}^{4} m_{3}^{9 / 2} \\
& +n_{2}^{3} m_{2}^{4} n_{3}^{9 / 2} m_{3}^{4}+n_{2}^{3} m_{2}^{4} n_{3}^{4} m_{3}^{9 / 2} .
\end{aligned}
$$

Herewith, from the perspective of state-space global invariants, we focus on the limiting nature of the underlying ensemble. Thus, we may choose the equal charge and anticharge limit by defining $m_{i}:=k$ and $n_{i}:=k$ for the calculation of the Ricci scalar. In this case, we find the following small negative curvature scalar:

$$
R(k)=-\frac{15}{16} \frac{1}{\pi k^{3 / 2}}
$$

Further, the physical meaning of taking an equal value of the charges and anticharges lies in the ensemble theory, namely, in the thermodynamic limit, all the statistical fluctuations of the charges and anticharges approach to a limiting Gaussian fluctuations. In this sense, we can take the average over the concerned individual Gaussian fluctuations. This shows that the limiting statistical ensemble of nonextremal nonlarge charge $D_{1}-D_{5}$ solutions yields an attractive statespace configuration. Finally, such a limiting procedure is indeed defined by considering the standard deviations of the equal integer charges and anticharges, and thus our interest in calculating the limiting Ricci scalar in order to know the nature of the long range interactions underlying in the system.

For a given entropy $S_{0}$, the constant entropy hypersurface is again some nonstandard curve:

$$
\left(\sqrt{n_{1}}+\sqrt{m_{1}}\right)\left(\sqrt{n_{2}}+\sqrt{m_{2}}\right)\left(\sqrt{n_{3}}+\sqrt{m_{3}}\right)=c,
$$

where the real constant $c$ takes the precise value of $S_{0} / 2 \pi$.

6.3. Eight Charge Configurations. From the perspective of the higher charged and anticharged black hole configurations in string theory, let us systematically analyze the underlying statistical structures. In this case, the state-space configuration of the nonextremal black hole involves finitely many nontrivially circularly fibered Kaluza-Klein monopoles. In this process, we enlist the complete set of nontrivial relative statespace correlation functions of the eight charged anticharged 
configurations, with respect to the lower parameter configurations, as considered in $[12,14]$. There have been calculations of the entropy of the extremal, near-extremal, and general nonextremal solutions in string theory; see, for instance, $[58,59]$. Inductively, the most general charge anticharge nonextremal black hole has the following entropy:

$$
S\left(n_{1}, m_{1}, n_{2}, m_{2}, n_{3}, m_{3}, n_{4}, m_{4}\right)=2 \pi \prod_{i=1}^{4}\left(\sqrt{n_{i}}+\sqrt{m_{i}}\right) .
$$

For the distinct $i, j, k \in\{1,2,3,4\}$, we find that the components of the metric tensor are

$$
\begin{aligned}
& g_{n_{i} n_{i}}=\frac{\pi}{2 n_{i}^{3 / 2}} \prod_{j \neq i}\left(\sqrt{n_{j}}+\sqrt{m_{j}}\right), \\
& g_{n_{i} n_{j}}=-\frac{\pi}{2\left(n_{i} n_{j}\right)^{1 / 2}} \prod_{i \neq k \neq j}\left(\sqrt{n_{k}}+\sqrt{m_{k}}\right) \text {, } \\
& g_{n_{i} m_{i}}=0 \text {, } \\
& g_{n_{i} m_{j}}=-\frac{\pi}{2\left(n_{i} m_{j}\right)^{1 / 2}} \prod_{i \neq k \neq j}\left(\sqrt{n_{k}}+\sqrt{m_{k}}\right) \text {, } \\
& g_{m_{i} m_{i}}=\frac{\pi}{2 m_{i}^{3 / 2}} \prod_{j \neq i}\left(\sqrt{n_{j}}+\sqrt{m_{j}}\right), \\
& g_{m_{i} m_{j}}=-\frac{\pi}{2\left(m_{i} m_{j}\right)^{1 / 2}} \prod_{i \neq k \neq j}\left(\sqrt{n_{k}}+\sqrt{m_{k}}\right) \text {. }
\end{aligned}
$$

From the above depiction, it is evident that the principle components of the state-space metric tensor $\left\{g_{n_{i} n_{i}}, g_{m_{i} m_{i}} \mid\right.$ $i=1,2,3,4\}$ essentially signify a set of definite heat capacities (or the related compressibilities) whose positivity in turn apprises that the black brane solutions comply with an underlying equilibrium statistical configuration. For an arbitrary number of the branes $\left\{n_{i}\right\}$ and antibranes $\left\{m_{i}\right\}$, we find that the associated state-space metric constraints as the diagonal pair correlation functions remain positive definite. In particular, $\forall i \in\{1,2,3,4\}$; it is clear that we have the following positivity conditions:

$$
g_{n_{i} n_{i}}>0\left|n_{i}, m_{i}>0, \quad g_{m_{i} m_{i}}>0\right| n_{i}, m_{i}>0 .
$$

As observed in $[12,14]$, we find that the ratios of diagonal components vary inversely with a multiple of a well-defined factor in the underlying parameters, namely, the charges and anticharges, which changes under the Gaussian fluctuations, whereas the ratios involving off diagonal components in effect uniquely inversely vary in the parameters of the chosen set $A_{i}$ of equilibrium black brane configurations. This suggests that the diagonal components weaken in a relatively controlled fashion into an equilibrium, in contrast with the off diagonal components, which vary over the domain of associated parameters defining the $D_{1}-D_{5}-P-K K$ nonextremal nonlarge charge configurations. In short, we can easily substantiate, for the distinct $x_{i}:=\left(n_{i}, m_{i}\right) \mid i \in\{1,2,3,4\}$ describing eight (anti)charge string theory black holes, that the relative pair correlation functions have distinct types of relative correlation functions. Apart from the zeros, infinities, and similar factorizations, we see that the nontrivial relative correlation functions satisfy the following scaling relations:

$$
\begin{aligned}
& \frac{g_{x_{i} x_{i}}}{g_{x_{j} x_{j}}}=\left(\frac{x_{j}}{x_{i}}\right)^{3 / 2} \frac{\sqrt{n_{j}}+\sqrt{m_{j}}}{\sqrt{n_{i}}+\sqrt{m_{i}}}, \\
& \frac{g_{x_{i} x_{j}}}{g_{x_{k} x_{l}}}=\left(\frac{x_{i} x_{j}}{x_{k} x_{l}}\right)^{-1 / 2} \frac{\prod_{i \neq p \neq j}\left(\sqrt{n_{p}}+\sqrt{m_{p}}\right)}{\prod_{k \neq q \neq l}\left(\sqrt{n_{q}}+\sqrt{m_{q}}\right)}, \\
& \frac{g_{x_{i} x_{i}}}{g_{x_{i} x_{k}}}=-\sqrt{\left(\frac{x_{k}}{x_{i}^{2}}\right)} \frac{\prod_{p \neq i}\left(\sqrt{n_{p}}+\sqrt{m_{p}}\right)}{\prod_{i \neq q \neq k}\left(\sqrt{n_{q}}+\sqrt{m_{q}}\right)} .
\end{aligned}
$$

As noticed in $[12,14]$, it is not difficult to analyze the statistical stability properties of the eight charged anticharged nonextremal black holes; namely, we can compute the principle minors associated with the state-space metric tensor and thereby argue that all the principle minors must be positive definite, in order to have a globally stable configuration. In the present case, it turns out that the above black hole is stable only when some of the charges and/or anticharges are held fixed or take specific values such that $p_{i}>0$ for all the dimensions of the state-space manifold. From the definition of the Hessian matrix of the associated entropy concerning the most general nonextremal nonlarge charged black holes, we observe that some of the principle minors $p_{i}$ are indeed nonpositive. In fact, we discover a uniform local stability criteria on the three-dimensional hypersurfaces, two-dimensional surface, and the one-dimensional line of the underlying state-space manifold. In order to simplify the factors of the higher principle, we may hereby collect the powers of each factor $\left(\sqrt{n_{i}}+\sqrt{m_{i}}\right)$ appearing in the expression of the entropy. With this notation, Appendix D provides the corresponding principle minors for the most general nonextremal nonlarge charged anticharged black hole in string theory involving finitely many nontrivially circularly fibered Kaluza-Klein monopoles.

Notice that the heat capacities, as the diagonal components $g_{i i}$, surface minor $p_{2}$, hypersurface minors $p_{3}, p_{5}$, $p_{6}$, and $p_{7}$, and the determinant of the state-space metric tensor, as the highest principle minor $p_{8}$ are examined as the functions of the number of branes $n$ and antibranes $m$. Thus, they describe the nature of the statistical fluctuations in the vacuum configuration. The corresponding scalar curvature is offered for an equal number of branes and antibranes $(n=m)$, which describes the nature of the long range statistical fluctuations. As per the above evaluation, we have obtained the exact expressions for the components of the metric tensor, principle minors, determinant of the metric tensor, and the underlying scalar curvature of the fluctuating statistical configuration of the eight parameter black holes in string theory. Qualitatively, the local and the global correlation properties of the limiting vacuum configuration can be realized under the statistical fluctuations. The first seven principle minors describe the local stability properties, and the last minor describes the global ensemble stability. 
The scalar curvature describes the corresponding phase space stability of the eight parameter black hole configuration. In general, there exists an akin higher degree polynomial equation on which the Ricci scalar curvature becomes null, and exactly on these points the state-space configuration of the underlying nonlarge charge nonextremal eight charge black hole system corresponds to a noninteracting statistical system. In this case, the corresponding state-space manifold $\left(M_{8}, g\right)$ becomes free from the statistical interaction with a vanishing state-space scalar curvature. As in case of the six charge configuration, we find interestingly that there exists an attractive configuration for the equal number of branes $n:=k$ and antibranes $m:=k$. In the limit of a large $k$, the corresponding system possesses a small negative value of the state-space scalar curvature:

$$
R(k)=-\frac{21}{32} \frac{1}{\pi k^{2}} .
$$

Interestingly, it turns out that the system becomes noninteracting in the limit of $k \rightarrow \infty$. For the case of the $n=k=m$, we observe that the corresponding principle minors reduce to the following constant values:

$$
\begin{aligned}
\left\{p_{i}\right\}_{i=1}^{8}=\{ & 4 \pi, 16 \pi^{2}, 32 \pi^{3}, 0,-2048 \pi^{5},-16384 \pi^{6}, \\
& \left.-163840 \pi^{7},-1048576 \pi^{8}\right\} .
\end{aligned}
$$

In this case, we find that the limiting underlying statistical system remains stable when at most three of the parameters, namely, $\left\{n_{i}=k=m_{i}\right\}$, are allowed to fluctuate. Herewith, we find for the case of $n:=k$ and $m:=k$ that the statespace manifold of the eight parameter brane and antibrane configuration is free from critical phenomena, except for the roots of the determinant. Thus, the regular state-space scalar curvature is comprehensively universal for the nonlarge charge nonextremal black brane configurations in string theory. In fact, the above perception turns out to be justified from the typical state-space geometry, namely, the definition of the metric tensor as the negative Hessian matrix of the duality invariant expression of the black brane entropy. In this case, we may nevertheless easily observe, for a given entropy $S_{0}$, that the constant entropy hypersurface is given by the following curve:

$$
\left(\sqrt{n_{1}}+\sqrt{m_{1}}\right)\left(\sqrt{n_{2}}+\sqrt{m_{2}}\right)\left(\sqrt{n_{3}}+\sqrt{m_{3}}\right)\left(\sqrt{n_{4}}+\sqrt{m_{4}}\right)=c,
$$

where $c$ is a real constant taking the precise value of $S_{0} / 2 \pi$. Under the vacuum fluctuations, the present analysis indicates that the entropy of the eight parameter black brane solution defines a nondegenerate embedding in the viewpoints of intrinsic state-space geometry. The above statespace computations determine an intricate set of statistical properties, namely, pair correlation functions and correlation volume, which reveal the possible nature of the associated parameters prescribing an ensemble of microstates of the dual conformal field theory living on the boundary of the black brane solution. For any black brane configuration, the above computation hereby shows that we can exhibit the state-space geometric acquisitions with an appropriate comprehension of the required parameters, for example, the charges and anticharges $\left\{n_{i}, m_{i}\right\}$, which define the coordinate charts. From the consideration of the state-space geometry, we have analyzed state-space pair correlation functions and the notion of stability of the most general nonextremal black hole in string theory. From the perspective of the intrinsic Riemannian geometry, we find that the stability of these black branes has been divulged from the positivity of principle minors of the space-state metric tensor.

Herewith, we have explicitly extended the state-space analysis for the four charge and four anticharge nonextremal black branes in string theory. The present consideration of the eight parameter black brane configurations, where the underlying leading order statistical entropy is written as a function of the charges $\left\{n_{i}\right\}$ and anticharges $\left\{m_{i}\right\}$, describes the stability properties under the Gaussian fluctuations. The present consideration includes all the special cases of the extremal and near-extremal configurations with a fewer number of charges and anticharges. In this case, we obtain the standard pattern of the underlying state-space geometry and constant entropy curve as that of the lower parameter nonextremal black holes. The local coordinate of the statespace manifold involves four charges and four anticharges of the underlying nonextremal black holes. In fact, the conclusion to be drawn remains the same, as the underlying state-space geometry remains well-defined as an intrinsic Riemannian manifold $N:=M_{8} \backslash \widetilde{B}$, where $\widetilde{B}$ is the set of roots of the determinant of the metric tensor. In particular, the state-space configuration of eight parameter black brane solutions remains nondegenerate for various domains of nonzero brane antibrane charges, except for the values, when the brane charges $\left\{n_{i}\right\}$ and antibrane charges $\left\{m_{i}\right\}$ belong to the set

$$
\begin{gathered}
\widetilde{B}:=\left\{\left(n_{1}, n_{2}, n_{3}, n_{4}, m_{1}, m_{2}, m_{3}, m_{4}\right) \mid\right. \\
\left.\left(n_{i}, m_{i}\right)=(0,0),(\infty, \infty)\right\},
\end{gathered}
$$

for a given brane-antibrane pair, $i \in\{1,2,3,4\}$. Our analysis indicates that the leading order statistical behavior of the black brane configurations in string theory remains intact under the inclusion of the Kaluza-Klein monopoles. In short, we have considered the eight charged anticharged string theory black brane configuration and analyzed the state-space pair correlation functions, relative scaling relations, stability conditions, and the corresponding global properties. Given a general nonextremal black brane configuration, we have exposed (i) for what conditions the considered black hole configuration is stable, (ii) how its state-space correlations scale in terms of the numbers of branes and antibranes.

\section{Conclusion and Outlook}

The Ruppenier geometry of two charge leading order extremal black holes remains flat or ill-defined. Thus, the statistical systems are, respectively, noninteracting or require higher derivative corrections. However, an addition of the third brane charge and other brane and antibrane charges 
indicates an interacting statistical system. The statistical fluctuations in the canonical ensemble lead to an interacting statistical system, as the scalar curvature of the state-space takes a nonzero value. We have explored the state-space geometric description of the charged extremal and associated charged anticharged nonextremal black holes in string theory.

Our analysis illustrates that the stability properties of the specific state-space hypersurface may exactly be exploited in general. The definite behavior of the state-space properties, as accounted in the specific cases, suggests that the underlying hypersurfaces of the state-space configuration include the intriguing mathematical feature. Namely, we find well-defined stability properties for the generic extremal and nonextremal black brane configurations, except for some specific values of the charges and anticharges. With and without the large charge limit, we have provided explicit forms of the higher principle minors of the state-space metric tensor for various charged, anticharged, extremal and nonextremal black holes in string theory. In this concern, the state-space configurations of the string theory black holes are generically well-defined and indicate an interacting statistical basis. Interestingly, we discover the state-space geometric nature of all possible general black brane configurations. From the very definition of the intrinsic metric tensor, the present analysis offers a definite stability character of string theory vacua.

Significantly, we notice that the related principle minors and the invariant state-space scalar curvature classify the underlying statistical fluctuations. The scalar curvature of a class of extremal black holes and the corresponding nonextremal black branes is everywhere regular with and without the stringy $\alpha^{\prime}$-corrections. A nonzero value of the state-space scalar curvature indicates an interacting underlying statistical system. We find that the antibrane corrections modify the state-space curvature, but do not induce phase transitions. In the limit of an extremal black hole, we construct the intrinsic geometric realization of a possible thermodynamic description at the zero temperature.

Importantly, the notion of the state-space of the considered black hole follows from the corresponding Wald and Cardy entropies. The microscopic and macroscopic entropies match in the large charge limit. From the perspective of statistical fluctuations, we anticipate the intrinsic geometric realization of two point local correlation functions and the corresponding global correlation length of the underlying conformal field theory configurations. In relation to the gauge-gravity correspondence and extremal black holes, our analysis describes state-space geometric properties of the corresponding boundary gauge theory.

General Remarks. For distinct $\{i, j\}$, the state-space pair correlations of an extremal configurations scale as

$$
\frac{g_{i i}}{g_{j j}}=\left(\frac{X_{j}}{X_{i}}\right)^{2}, \quad \frac{g_{i j}}{g_{i i}}=-\frac{X_{i}}{X_{j}} .
$$

In general, the black brane configurations in string theory can be categorized as per their state-space invariants. The underlying subconfigurations turn out to be welldefined over possible domains, whenever there exists a respective set of nonzero state-space principle minors. The underlying full configuration turns out to be everywhere well-defined, whenever there exists a nonzero state-space determinant. The underlying configuration corresponds to an interacting statistical system, whenever there exists a nonzero state-space scalar curvature. The intrinsic statespace manifold of extremal/nonextremal and supersymmetric/nonsupersymmetric string theory black holes may intrinsically be described by an embedding:

$$
\left(M_{(n)}, g\right) \hookrightarrow\left(M_{(n+1)}, \widetilde{g}\right) .
$$

The extremal state-space configuration may be examined as a restriction to the full counting entropy with an intrinsic state-space metric tensor $\left.g \mapsto \tilde{g}\right|_{r_{+}=r_{-}}$. Furthermore, the state-space configurations of the supersymmetric black holes may be examined as the BPS restriction of the full space of the counting entropy with an understanding that the intrinsic state-space metric tensor is defined as $g:=\left.\tilde{g}\right|_{M=M_{0}}$. From the perspective of string theory, the restrictions $r_{+}=$ $r_{-}$and $M=M_{0}\left(P_{i}, Q_{i}\right)$ should be understood as the fact that it has been applied to an assigned entropy of the nonextremal/nonsupersymmetric (or nearly extremal/nearly supersymmetric) black brane configuration. This allows one to compute the fluctuations in ADM mass of the black hole. In the viewpoint of the present research on the state-space geometry, it is worth mentioning that the dependence of the mass to the entropy of a nonextremal black hole comes from the contribution of the antibranes, see, for instance, Section 4.5, and so we may examine the corresponding Weinhold chemical geometry, as mentioned in Section 4.3.

Future Directions and Open Issues. The state-space instabilities and their relation to the dual microscopic conformal field theories could open up a number of new realizations. The state-space perspective includes the following issues.

(i) Multicenter Gibbons-Hawking solutions [69, 70] with generalized base space manifolds having a mixing of positive and negative residues, see [71, 72] for a perspective development of state-space geometry by invoking the role of foaming of black holes and plumbing the Abyss for the microstates counting of black rings.

(ii) Dual conformal field theories and string duality symmetries, see [61] for a quantum mechanical perspective of superconformal black holes and $[73,74]$ for the origin of gravitational thermodynamics and the role of giant gravitons in conformal field theory.

(iii) Stabilization against local and/or global perturbations, see [75-80] for black brane dynamics, stability, and critical phenomena. Thus, the consideration of state-space geometry is well-suited for examining the domain of instability. This includes Gregory-Laflamme (GL) modes, chemical potential 
fluctuations, electric-magnetic charges and dipole charges, rotational fluctuations, and the thermodynamic temperature fluctuations for the near-extremal and nonextremal black brane solutions. We leave this perspective of the state-space geometry open for a future research.

In general, various $D$ dimensional black brane configurations, see, for instance, [75-80] for black rings in $D>5$ spacetime dimensions with $S^{1} \times S^{D-3}$ horizon topology, and the higher horizon topologies, for example, $S^{1} \times S^{1} \times S^{2}, S^{3} \times S^{3}$, and so forth offer a platform to extend the consideration of the statespace geometry.

On the other hand, the bubbling black brane solutions, namely, Lin, Lunin, and Maldacena (LLM) geometries [81], are interesting from the perspective of Mathur's Fuzzball conjecture(s). From the perspective of the generalized hyperKähler manifolds, Mathur's conjecture [82-85] reduces to classifying and counting asymptotically flat four-dimensional hyper Kähler manifolds [71] which have moduli regions of uniform signature $(+,+,+,+)$ and $(-,-,-,-)$.

Finally, the new physics at the length of the Planck scale anticipates an analysis of the state-space configurations. In particular, it materializes that the state-space geometry may be explored with the parameters of the foam geometries [71], and the corresponding empty space virtual black holes, see [81] for the notion of bubbling AdS space and 1/2 BPS geometries. In such cases, the local and global statistical correlations, among the parameters of the microstates of the black hole conformal field theory $[60,61]$, would involve the foams of two spheres. From the perspective of the string theory, the present exploration thus opens up an avenue for learning new insights into the promising structures of the black brane space-time configurations at very small scales.

\section{Appendices}

In these appendices, we provide explicit forms of the statespace correlation arising from the metric tensor of the charged (non)extremal (non)large black holes in string theory. In fact, our analysis illustrates that the stability properties of the specific state-space hypersurface may exactly be exploited in general. The definite behavior of state-space properties, as accounted in the concerned main sections, suggests that the various intriguing hypersurfaces of the statespace configuration include the nice feature that they do have definite stability properties, except for some specific values of the charges and anticharges.

As mentioned in the main sections, these configurations are generically well-defined and indicate an interacting statistical basis. Herewith, we discover that the statespace geometry of the general black brane configurations in string theory indicates the possible nature of the underlying statistical fluctuations. Significantly, we notice from the very definition of the intrinsic metric tensor that the related statistical pair correlation functions and relative statistical correlation functions take the following exact expressions.

\section{A. Correlations for Three Charge Configurations}

Following the notion of the fluctuations, we see from the Hessian of the entropy equation (30) that the components of state-space metric tensor are

$$
\begin{array}{ll}
g_{n_{1} n_{1}}=\frac{\pi}{2 n_{1}} \sqrt{\frac{n_{5} n_{p}}{n_{1}}}, \quad g_{n_{1} n_{5}}=-\frac{\pi}{2} \sqrt{\frac{n_{p}}{n_{1} n_{5}}}, \\
g_{n_{1} n_{p}}=-\frac{\pi}{2} \sqrt{\frac{n_{5}}{n_{1} n_{p}}}, \quad g_{n_{5} n_{5}}=\frac{\pi}{2 n_{5}} \sqrt{\frac{n_{1} n_{p}}{n_{5}}}, \\
g_{n_{5} n_{p}}=-\frac{\pi}{2} \sqrt{\frac{n_{1}}{n_{5} n_{p}}}, \quad g_{n_{p} n_{p}}=\frac{\pi}{2 n_{p}} \sqrt{\frac{n_{1} n_{5}}{n_{p}}} .
\end{array}
$$

For distinct $i, j \in\{1,5\}$ and $p$, the list of relative correlation functions follows the scaling relations:

$$
\begin{aligned}
\frac{g_{i i}}{g_{j j}}=\left(\frac{n_{j}}{n_{i}}\right)^{2}, & \frac{g_{i i}}{g_{p p}}=\left(\frac{n_{p}}{n_{i}}\right)^{2}, & \frac{g_{i i}}{g_{i j}}=-\left(\frac{n_{j}}{n_{i}}\right), \\
\frac{g_{i i}}{g_{i p}}=-\left(\frac{n_{p}}{n_{i}}\right), & \frac{g_{i p}}{g_{j p}}=\left(\frac{n_{j}}{n_{i}}\right), & \frac{g_{i i}}{g_{j p}}=-\left(\frac{n_{j} n_{p}}{n_{i}^{2}}\right), \\
\frac{g_{i p}}{g_{p p}}=-\left(\frac{n_{p}}{n_{i}}\right), & \frac{g_{i j}}{g_{i p}}=\left(\frac{n_{p}}{n_{j}}\right), & \frac{g_{i j}}{g_{p p}}=-\left(\frac{n_{p}^{2}}{n_{i} n_{j}}\right) .
\end{aligned}
$$

\section{B. Correlations for Four Charge Configurations}

For the given entropy as in (36), we find that the components of the metric tensor are

$$
\begin{aligned}
& g_{n_{1} n_{1}}=\frac{\pi}{2} \sqrt{\frac{n_{5}}{n_{1}^{3}}}\left(\sqrt{n_{p}}+\sqrt{\overline{n_{p}}}\right), \\
& g_{n_{1} n_{5}}=-\frac{\pi}{2 \sqrt{n_{1} n_{5}}}\left(\sqrt{n_{p}}+\sqrt{\overline{n_{p}}}\right), \\
& g_{n_{1} n_{p}}=-\frac{\pi}{2} \sqrt{\frac{n_{5}}{n_{1} n_{p}}}, \quad g_{n_{1} \overline{n_{p}}}=-\frac{\pi}{2} \sqrt{\frac{n_{5}}{n_{1} \overline{n_{p}}}}, \\
& g_{n_{5} n_{5}}=\frac{\pi}{2} \sqrt{\frac{n_{1}}{n_{5}^{3}}}\left(\sqrt{n_{p}}+\sqrt{\overline{n_{p}}}\right), \quad g_{n_{5} n_{p}}=-\frac{\pi}{2} \sqrt{\frac{n_{1}}{n_{5} n_{p}}}, \\
& g_{n_{5} \bar{n}_{p}}=-\frac{\pi}{2} \sqrt{\frac{n_{1}}{n_{5} \overline{n_{p}}}}, \quad g_{n_{p} n_{p}}=\frac{\pi}{2} \sqrt{\frac{n_{1} n_{5}}{n_{p}^{3}}}, \\
& g_{n_{p} \overline{n_{p}}}=0, \\
& g_{\overline{n_{p} n_{p}}}=\frac{\pi}{2} \sqrt{\frac{n_{1} n_{5}}{\bar{n}_{p}^{3}}} .
\end{aligned}
$$


For distinct $i, j \in\{1,5\}$, and $k, l \in\{p, \bar{p}\}$ describing four charge nonextremal $D_{1}-D_{5}-P-\bar{P}$ black holes, the statistical pair correlations consist of the following scaling relations:

$$
\begin{aligned}
& \frac{g_{i i}}{g_{j j}}=\left(\frac{n_{j}}{n_{i}}\right)^{2}, \quad \frac{g_{i i}}{g_{k k}}=\frac{n_{k}}{n_{i}^{2}} \sqrt{n_{k}}\left(\sqrt{n_{p}}+\sqrt{\overline{n_{p}}}\right), \\
& \frac{g_{i i}}{g_{i j}}=-\frac{n_{j}}{n_{i}}, \\
& \frac{g_{i i}}{g_{i k}}=-\frac{\sqrt{n_{k}}}{n_{i}}\left(\sqrt{n_{p}}+\sqrt{\overline{n_{p}}}\right), \\
& \frac{g_{i k}}{g_{j k}}=\frac{n_{j}}{n_{i}}, \quad \frac{g_{i i}}{g_{j k}}=-\frac{n_{j}}{n_{i}^{2}} \sqrt{n_{k}}\left(\sqrt{n_{p}}+\sqrt{\overline{n_{p}}}\right), \\
& \frac{g_{i k}}{g_{k k}}=-\frac{n_{k}}{n_{i}}, \quad \frac{g_{i j}}{g_{i k}}=\frac{\sqrt{n_{k}}}{n_{j}}\left(\sqrt{n_{p}}+\sqrt{\overline{n_{p}}}\right), \\
& \frac{g_{i j}}{g_{k k}}=-\frac{n_{k}}{n_{i} n_{j}} \sqrt{n_{k}}\left(\sqrt{n_{p}}+\sqrt{\overline{n_{p}}}\right) .
\end{aligned}
$$

Notice that the list of other mixed relative correlation functions concerning the nonextremal $D_{1}-D_{5}-P-\bar{P}$ black holes read as

$$
\begin{array}{llll}
\frac{g_{i k}}{g_{i l}}=\sqrt{\frac{n_{l}}{n_{k}}}, & \frac{g_{i k}}{g_{j l}}=\frac{n_{j}}{n_{i}} \sqrt{\frac{n_{l}}{n_{k}}}, & & \frac{g_{k l}}{g_{i j}}=0, \\
\frac{g_{k l}}{g_{i i}}=0, & \frac{g_{k k}}{g_{l l}}=\left(\frac{n_{l}}{n_{k}}\right)^{3 / 2}, & \frac{g_{k l}}{g_{k k}}=0 .
\end{array}
$$

\section{Correlations for Six Charge Configurations}

Over generic nonlarge charge domains, we find from the entropy equation (46) that the components of the covariant state-space metric tensor are given by the following expressions:

$$
\begin{aligned}
& g_{n_{1} n_{1}}=\frac{\pi}{2 n_{1}^{3 / 2}}\left(\sqrt{n_{2}}+\sqrt{m_{2}}\right)\left(\sqrt{n_{3}}+\sqrt{m_{3}}\right), \quad g_{n_{1} m_{1}}=0, \\
& g_{n_{1} n_{2}}=-\frac{\pi}{2 \sqrt{n_{1} n_{2}}}\left(\sqrt{n_{3}}+\sqrt{m_{3}}\right), \\
& g_{n_{1} m_{2}}=-\frac{\pi}{2 \sqrt{n_{1} m_{2}}}\left(\sqrt{n_{3}}+\sqrt{m_{3}}\right), \\
& g_{n_{1} n_{3}}=-\frac{\pi}{2 \sqrt{n_{1} n_{3}}}\left(\sqrt{n_{2}}+\sqrt{m_{2}}\right), \\
& g_{n_{1} m_{3}}=-\frac{\pi}{2 \sqrt{n_{1} m_{3}}}\left(\sqrt{n_{2}}+\sqrt{m_{2}}\right),
\end{aligned}
$$

$$
\begin{aligned}
& g_{m_{1} m_{1}}=\frac{\pi}{2 m_{1}^{3 / 2}}\left(\sqrt{n_{2}}+\sqrt{m_{2}}\right)\left(\sqrt{n_{3}}+\sqrt{m_{3}}\right), \\
& g_{m_{1} n_{2}}=-\frac{\pi}{2 \sqrt{m_{1} n_{2}}}\left(\sqrt{n_{3}}+\sqrt{m_{3}}\right), \\
& g_{m_{1} m_{2}}=-\frac{\pi}{2 \sqrt{m_{1} m_{2}}}\left(\sqrt{n_{3}}+\sqrt{m_{3}}\right), \\
& g_{m_{1} n_{3}}=-\frac{\pi}{2 \sqrt{m_{1} n_{3}}}\left(\sqrt{n_{2}}+\sqrt{m_{2}}\right), \\
& g_{m_{1} m_{3}}=-\frac{\pi}{2 \sqrt{m_{1} m_{3}}}\left(\sqrt{n_{2}}+\sqrt{m_{2}}\right), \\
& g_{n_{2} n_{2}}=\frac{\pi}{2 n_{2}^{3 / 2}}\left(\sqrt{n_{1}}+\sqrt{m_{1}}\right)\left(\sqrt{n_{3}}+\sqrt{m_{3}}\right), \\
& g_{n_{2} m_{2}}=0, \\
& g_{n_{3} n_{3}}=\frac{\pi}{2 n_{3}^{3 / 2}}\left(\sqrt{n_{1}}+\sqrt{m_{1}}\right)\left(\sqrt{n_{2} n_{3}}+\sqrt{m_{2}}\right), \quad \frac{\pi}{2 m_{3}^{3 / 2}}\left(\sqrt{n_{2} n_{3}}+\sqrt{m_{1}}+\sqrt{m_{1} m_{3}}\right)\left(\sqrt{n_{2}}+\sqrt{m_{2}}\right) . \\
& g_{n_{2} m_{3}}=-\frac{\pi}{2 \sqrt{n_{2} m_{3}}}\left(\sqrt{n_{1}}+\sqrt{m_{1}}\right), \\
& g_{m_{2} m_{2}}=\frac{\pi}{2 m_{2}^{3 / 2}}\left(\sqrt{n_{1}}+\sqrt{m_{1}}\right)\left(\sqrt{n_{3}}+\sqrt{m_{3}}\right), \\
& g_{2}=-\frac{\pi}{2 \sqrt{m_{2} m_{3}}}\left(\sqrt{n_{1}}+\sqrt{m_{1}}\right),
\end{aligned}
$$

In this case, from the definition of the relative statistical correlation functions, for $i, j \in\left\{n_{1}, m_{1}\right\}$, and $k, l \in\left\{n_{2}, m_{2}\right\}$, the relative correlation functions satisfy the following scaling relations:

$$
\begin{aligned}
& \frac{g_{i i}}{g_{j j}}=\left(\frac{j}{i}\right)^{3 / 2}, \quad \frac{g_{i i}}{g_{k k}}=\left(\frac{k}{i}\right)^{3 / 2}\left(\frac{\sqrt{n_{2}}+\sqrt{m_{2}}}{\sqrt{n_{3}}+\sqrt{m_{3}}}\right), \\
& \frac{g_{i j}}{g_{i i}}=0, \\
& \frac{g_{i i}}{g_{i k}}=-\frac{\sqrt{k}}{i}\left(\sqrt{n_{2}}+\sqrt{m_{2}}\right), \quad \frac{g_{i k}}{g_{j k}}=\sqrt{\frac{j}{i}}, \\
& \frac{g_{i i}}{g_{j k}}=-\frac{\sqrt{j k}}{i^{3 / 2}}\left(\sqrt{n_{2}}+\sqrt{m_{2}}\right), \quad \\
& \frac{g_{k k}}{g_{i k}}=-\frac{\sqrt{i}}{k}\left(\sqrt{n_{2}}+\sqrt{m_{2}}\right), \quad \frac{g_{i j}}{g_{i k}}=0, \quad \frac{g_{i j}}{g_{k k}}=0 .
\end{aligned}
$$


The other concerned relative correlation functions are

$$
\begin{array}{rlrl}
\frac{g_{i k}}{g_{i l}} & =\sqrt{\frac{l}{k}}, & \frac{g_{i k}}{g_{j l}}=\sqrt{\frac{j l}{i k}}, & \frac{g_{i j}}{g_{k l}}=\text { n.d., } \\
\frac{g_{k l}}{g_{i i}}=0, & \frac{g_{k k}}{g_{l l}}=\left(\frac{l}{k}\right)^{3 / 2}, & \frac{g_{k l}}{g_{k k}}=0 .
\end{array}
$$

For $k, l \in\left\{n_{2}, m_{2}\right\}$, and $m, n \in\left\{n_{3}, m_{3}\right\}$, we have

$$
\begin{aligned}
& \frac{g_{k k}}{g_{m m}}=\left(\frac{m}{k}\right)^{3 / 2}\left(\frac{\sqrt{n_{3}}+\sqrt{m_{3}}}{\sqrt{n_{2}}+\sqrt{m_{2}}}\right), \\
& \frac{g_{k l}}{g_{k k}}=0, \quad \frac{g_{k k}}{g_{k m}}=-\frac{\sqrt{m}}{k}\left(\sqrt{n_{3}}+\sqrt{m_{3}}\right), \\
& \frac{g_{k m}}{g_{l m}}=\sqrt{\frac{l}{k}}, \quad \frac{g_{k k}}{g_{l m}}=-\frac{\sqrt{l m}}{k^{3 / 2}}\left(\sqrt{n_{3}}+\sqrt{m_{3}}\right), \\
& \frac{g_{m m}}{g_{k m}}=-\frac{\sqrt{k}}{m}\left(\sqrt{n_{2}}+\sqrt{m_{2}}\right), \\
& \frac{g_{k l}}{g_{k m}}=0, \quad \frac{g_{k l}}{g_{m m}}=0 .
\end{aligned}
$$

The other concerned relative correlation functions are

$$
\begin{array}{rlrlrl}
\frac{g_{k m}}{g_{k n}} & =\sqrt{\frac{n}{m}}, & \frac{g_{k m}}{g_{l n}}=\sqrt{\frac{l n}{k m}}, & & \frac{g_{k l}}{g_{m n}}=\text { n.d., } \\
\frac{g_{m n}}{g_{k k}}=0, & \frac{g_{m m}}{g_{n n}}=\left(\frac{n}{m}\right)^{3 / 2}, & \frac{g_{m n}}{g_{m m}} & =0 .
\end{array}
$$

However, for $i, j \in\left\{n_{1}, m_{1}\right\}$, and $m, n \in\left\{n_{3}, m_{3}\right\}$, we have

$$
\begin{aligned}
\frac{g_{i i}}{g_{m m}} & =\left(\frac{m}{i}\right)^{3 / 2}\left(\frac{\sqrt{n_{3}}+\sqrt{m_{3}}}{\sqrt{n_{1}}+\sqrt{m_{1}}}\right), \quad \frac{g_{i j}}{g_{i i}}=0, \\
\frac{g_{i i}}{g_{i m}} & =-\frac{\sqrt{m}}{i}\left(\sqrt{n_{3}}+\sqrt{m_{3}}\right), \\
\frac{g_{i m}}{g_{j m}} & =\sqrt{\frac{j}{i}}, \quad \frac{g_{i i}}{g_{j m}}=-\frac{\sqrt{j m}}{i^{3 / 2}}\left(\sqrt{n_{3}}+\sqrt{m_{3}}\right), \\
\frac{g_{m m}}{g_{i m}} & =-\frac{\sqrt{i}}{m}\left(\sqrt{n_{1}}+\sqrt{m_{1}}\right), \\
\frac{g_{i j}}{g_{i m}} & =0, \quad \frac{g_{i j}}{g_{m m}}=0, \quad \frac{g_{i m}}{g_{i n}}=\sqrt{\frac{n}{m}}, \\
\frac{g_{i m}}{g_{j n}} & =\sqrt{\frac{j n}{i m}}, \quad \frac{g_{i j}}{g_{m n}}=\text { n.d., } \\
\frac{g_{m n}}{g_{i i}} & =0, \quad \frac{g_{m n}}{g_{m m}}=0 .
\end{aligned}
$$

\section{Principle Minors for Eight Charge Configurations}

For the entropy equation (58) of the most general nonextremal nonlarge charged anticharged black hole in string involving finitely many nontrivially circularly fibered KaluzaKlein monopoles, the principle minors take the following expressions:

$$
\begin{aligned}
& p_{1}=\frac{\pi}{2 n_{1}^{3 / 2}}\left(\sqrt{n_{2}}+\sqrt{m_{2}}\right)\left(\sqrt{n_{3}}+\sqrt{m_{3}}\right)\left(\sqrt{n_{4}}+\sqrt{m_{4}}\right), \\
& p_{2}=\frac{\pi^{2}}{4\left(n_{1} m_{1}\right)^{3 / 2}}\left(\sqrt{n_{2}}+\sqrt{m_{2}}\right)^{2}\left(\sqrt{n_{3}}+\sqrt{m_{3}}\right)^{2} \\
& \times\left(\sqrt{n_{4}}+\sqrt{m_{4}}\right)^{2}, \\
& p_{3}=\frac{\pi^{3}}{8\left(n_{1} m_{1} n_{2}\right)^{3 / 2}}\left(\sqrt{n_{3}}+\sqrt{m_{3}}\right)^{3}\left(\sqrt{n_{4}}+\sqrt{m_{4}}\right)^{3} \\
& \times\left(\sqrt{n_{2}}+\sqrt{m_{2}}\right) \sqrt{m_{2}}\left(\sqrt{n_{1}}+\sqrt{m_{1}}\right), \\
& p_{4}=0 \text {, } \\
& p_{5}=-\frac{\pi^{5}}{8\left(n_{1} n_{2} m_{2} m_{1}\right)^{3 / 2} n_{3}}\left(\sqrt{n_{2}}+\sqrt{m_{2}}\right)^{3} \\
& \times\left(\sqrt{n_{3}}+\sqrt{m_{3}}\right)^{3}\left(\sqrt{n_{4}}+\sqrt{m_{4}}\right)^{5}\left(\sqrt{n_{1}}+\sqrt{m_{1}}\right)^{3}, \\
& p_{6}=-\frac{\pi^{6}}{16\left(n_{1} n_{2} m_{1} m_{2} n_{3} m_{3}\right)^{3 / 2}}\left(\sqrt{n_{2}}+\sqrt{m_{2}}\right)^{4} \\
& \times\left(\sqrt{n_{3}}+\sqrt{m_{3}}\right)^{4}\left(\sqrt{n_{4}}+\sqrt{m_{4}}\right)^{6}\left(\sqrt{n_{1}}+\sqrt{m_{1}}\right)^{4}, \\
& p_{7}=-\frac{\pi^{7}}{32\left(n_{1} m_{1} n_{2} m_{2} n_{3} m_{3} n_{4}\right)^{3 / 2}}\left(\sqrt{n_{2}}+\sqrt{m_{2}}\right)^{5} \\
& \times\left(\sqrt{n_{3}}+\sqrt{m_{3}}\right)^{5}\left(\sqrt{n_{4}}+\sqrt{m_{4}}\right)^{5}\left(4 \sqrt{n_{4}}+\sqrt{m_{4}}\right) \\
& \times\left(\sqrt{n_{1}}+\sqrt{m_{1}}\right)^{5}, \\
& p_{8}=-\frac{\pi^{8}}{16\left(\prod_{i=1}^{4} n_{i} m_{i}\right)^{3 / 2}}\left(\sqrt{n_{2}}+\sqrt{m_{2}}\right)^{6} \\
& \times\left(\sqrt{n_{3}}+\sqrt{m_{3}}\right)^{6}\left(\sqrt{n_{4}}+\sqrt{m_{4}}\right)^{6}\left(\sqrt{n_{1}}+\sqrt{m_{1}}\right)^{6} .
\end{aligned}
$$

\section{Conflict of Interests}

The author declares that there is no conflict of interests regarding the publication of this paper.

\section{Acknowledgments}

This work has been supported in part by the European Research Council Grant no. 226455, "SUPERSYMMETRY, QUANTUM GRAVITY AND GAUGE FIELDS (SUPERFIELDS)." Bhupendra Nath Tiwari would like to thank Prof. V. Ravishankar for his support and encouragements towards the research in string theory. This work was conducted during 
the period Bhupendra Nath Tiwari served as a postdoctoral research fellow at the INFN-Laboratori Nazionali di Frascati, Roma, Italy.

\section{References}

[1] F. Weinhold, "Metric geometry of equilibrium thermodynamics," The Journal of Chemical Physics, vol. 63, no. 6, pp. 24792483, 1975.

[2] F. Weinhold, "Metric geometry of equilibrium thermodynamics. II. Scaling, homogeneity, and generalized Gibbs-Duhem relations," The Journal of Chemical Physics, vol. 63, no. 6, pp. 2484-2487, 1975.

[3] G. Ruppeiner, "Thermodynamics: a Riemannian geometric model," Physical Review A, vol. 20, no. 4, pp. 1608-1613, 1979.

[4] G. Ruppeiner, "Thermodynamic critical fluctuation theory?" Physical Review Letters, vol. 50, no. 5, pp. 287-290, 1983.

[5] G. Ruppeiner, "New thermodynamic fluctuation theory using path integrals," Physical Review A, vol. 27, no. 2, pp. 1116-1133, 1983.

[6] G. Ruppeiner, "Riemannian geometry in thermodynamic fluctuation theory," Reviews of Modern Physics, vol. 67, p. 605, 1995, Erratum in "Riemannian geometry in thermodynamic fluctuation theory”, Reviews of Modern Physics, vol. 68, pp. 313, 1996.

[7] G. Ruppeiner and C. Davis, "Thermodynamic curvature of the multicomponent ideal gas," Physical Review A, vol. 41, no. 4, pp. 2200-2202, 1990.

[8] G. Ruppeiner, "Stability and fluctuations in black hole thermodynamics," Physical Review D, vol. 75, no. 2, Article ID 024037, 2007.

[9] G. Ruppeiner, "Thermodynamic curvature and phase transitions in Kerr-Newman black holes," Physical Review D, vol. 78, no. 2, Article ID 024016, 2008.

[10] B. N. Tiwari, Éditions Universitaires Européennes, Germany, 2001, New Paths Towards Quantum Gravity, Holbaek, Denmark, 2008, http://arxiv.org/abs/0801.4087.

[11] T. Sarkar, G. Sengupta, and B. N. Tiwari, "Thermodynamic geometry and extremal black holes in string theory," Journal of High Energy Physics, vol. 2008, no. 10, article 076, 2008.

[12] S. Bellucci and B. N. Tiwari, "On the microscopic perspective of black branes thermodynamic geometry," Entropy, vol. 12, no. 10, pp. 2097-2143, 2010.

[13] T. Sarkar, G. Sengupta, and B. N. Tiwari, "On the thermodynamic geometry of BTZ black holes," Journal of High Energy Physics, vol. 2006, no. 11, article 015, 2006.

[14] S. Bellucci and B. N. Tiwari, "State-space correlations and stabilities," Physical Review D, vol. 82, Article ID 084008, 2010.

[15] S. Bellucci and B. N. Tiwari, "Thermodynamic geometry and Hawking radiation," Journal of High Energy Physics, vol. 2010, article 30, 2010.

[16] S. Bellucci and B. N. Tiwari, "An exact fluctuating 1/2-BPS configuration," Journal of High Energy Physics, no. 5, article 023, 2010.

[17] S. Bellucci and B. N. Tiwari, "State-space manifold and rotating black holes," Journal of High Energy Physics, no. 1, article 118, 2011.

[18] S. Bellucci and B. N. Tiwari, "Black strings, black rings and statespace manifold," International Journal of Modern Physics A, vol. 26, no. 32, pp. 5403-5464, 2011.
[19] S. Bellucci and B. N. Tiwari, "State-space geometry, nonextremal black holes and Kaluza-Klein monopoles," Modern Physics Letters A, vol. 27, no. 16, Article ID 1250095, 2012.

[20] J. E. Åman, I. Bengtsson, and N. Pidokrajt, "Flat information geometries in black hole thermodynamics," General Relativity and Gravitation, vol. 38, no. 8, pp. 1305-1315, 2006.

[21] J. E. Åman, I. Bengtsson, and N. Pidokrajt, "Geometry of black hole thermodynamics," General Relativity and Gravitation, vol. 35, no. 10, pp. 1733-1743, 2003.

[22] J. E. Åman and N. Pidokrajt, "Geometry of higher-dimensional black hole thermodynamics," Physical Review D, vol. 73, no. 2, Article ID 024017, 2006.

[23] J. E. Aman, J. Bedford, D. Grumiller, N. Pidokrajt, and J. Ward, "Ruppeiner theory of black hole thermodynamics," Journal of Physics: Conference Series, vol. 66, no. 10, Article ID 012007, 2003.

[24] G. Arcioni and E. Lozano-Tellechea, "Stability and critical phenomena of black holes and black rings," Physical Review D, vol. 72, no. 10, Article ID 104021, 2005.

[25] J. Y. Shen, R. G. Cai, B. Wang, and R. K. Su, “Thermodynamic geometry and critical behavior of black holes," International Journal of Modern Physics A, vol. 22, no. 11, 2007.

[26] M. Santoro and A. S. Benight, "On the geometrical thermodynamics of chemical reactions," http://arxiv.org/abs/math-ph/ 0507026.

[27] B. N. Tiwari, "New Paths Towards Quantum Gravity," Holbaek, Denmark, 2008.

[28] S. Bellucci, V. Chandra, and B. N. Tiwari, "On the thermodynamic geometry of hot QCD," International Journal of Modern Physics A, vol. 26, no. 43, 2011.

[29] S. Bellucci, V. Chandra, and B. N. Tiwari, "Thermodynamic geometric stability of Quarkonia states," International Journal of Modern Physics A, vol. 26, no. 2665, 2011.

[30] E. Witten, "String theory dynamics in various dimensions," Nuclear Physics B, vol. 443, no. 1-2, pp. 85-126, 1995.

[31] S. Ferrara, R. Kallosh, and A. Strominger, " $N=2$ extremal black holes," Physical Review D, vol. 52, no. 10, pp. R5412-R5416, 1995.

[32] A. Strominger, "Macroscopic entropy of $N=2$ extremal black holes," Physics Letters B, vol. 383, no. 1, pp. 39-43, 1996.

[33] S. Ferrara and R. Kallosh, "Supersymmetry and attractors," Physical Review D, vol. 54, no. 2, pp. 1514-1524, 1996.

[34] S. Ferrara, G. W. Gibbons, and R. Kallosh, "Black holes and critical points in moduli space," Nuclear Physics B, vol. 500, no. 1-3, pp. 75-93, 1997.

[35] S. Bellucci, S. Ferrara, and A. Marrani, "On some properties of the attractor equations," Physics Letters B, vol. 635, no. 2-3, pp. 172-179, 2006.

[36] S. Bellucci, S. Ferrara, and A. Marrani, Supersymmetric Mechanics: The Attractor Mechanism and Space-Time Singularities, vol. 2 of Lecture Notes in Physics, Springer, Heidelberg, Germany, 2006.

[37] S. Bellucci, S. Ferrara, M. Günaydin, and A. Marrani, "Charge orbits of symmetric special geometries and attractors," International Journal of Modern Physics A, vol. 21, no. 25, Article ID 5043, 2006.

[38] S. Bellucci, S. Ferrara, A. Marrani, and A. Yeranyan, "Mirror Fermat Calabi-Yau threefolds and Landau-Ginzburg black-hole attractors," Rivista del Nuovo Cimento, vol. 29, no. 5, pp. 1-88, 2006. 
[39] S. Bellucci, S. Ferrara, and A. Marrani, Contribution to the Proceedings of the XVII SIGRAV Conference, Turin, Italy, September 2006.

[40] S. Bellucci, S. Ferrara, R. Kallosh, and A. Marrani, "Extremal black hole and flux vacua attractors," in Supersymmetric Mechanics, vol. 755 of Lecture Notes in Physics, pp. 115-191, Springer, Heidelberg, Germany, 2008.

[41] S. Bellucci, S. Ferrara, and A. Marrani, "Attractors in black," Fortschritte der Physik, vol. 56, no. 7-9, pp. 761-785, 2008.

[42] S. Bellucci, S. Ferrara, M. Günaydin, and A. Marrani, "SAM lectures on extremal black holes in $\mathrm{d}=4$ extended supergravity," http://arxiv.org/abs/0905.3739.

[43] A. Sen, "Stretching the horizon of a higher dimensional small black hole," Journal of High Energy Physics, no. 7, article 073, 2005.

[44] A. Sen, "How does a fundamental string stretch its horizon?" Journal of High Energy Physics, no. 5, article 059, 2005.

[45] A. Dabholkar, "Exact counting of supersymmetric black hole microstates," Physical Review Letters, vol. 94, no. 24, Article ID 241301, 2005.

[46] A. Dabholkar, F. Denef, G. W. Moore, and B. Pioline, "Precision counting of small black holes," Journal of High Energy Physics, no. 10, article 096, 2005.

[47] A. Dabholkar, F. Denef, G. W. Moore, and B. Pioline, "Exact and asymptotic degeneracies of small black holes," Journal of High Energy Physics, no. 8, article 021, 2005.

[48] A. Sen, "Black holes and the spectrum of half-BPS states in $N=4$ supersymmetric string theory," Advances in Theoretical and Mathematical Physics, vol. 9, no. 4, pp. 527-558, 2005.

[49] B. D. Chowdhury and S. D. Mathur, "Fractional brane state in the early universe," 2006, http://arxiv.org/abs/hep-th/0611330.

[50] A. Ghodsi and M. R. Garousi, "Entropy function for nonextremal D1D5 and D2D6NS5-branes," Journal of High Energy Physics, no. 10, article 036, 2007.

[51] R.-G. Cai and D.-W. Pang, "Entropy function for non-extremal black holes in string theory," Journal of High Energy Physics, no. 5, article 023, 2007.

[52] M. R. Garousi and A. Ghodsi, "On attractor mechanism and entropy function for non-extremal black holes/branes," Journal of High Energy Physics, no. 5, article 043, 2007.

[53] A. Strominger and C. Vafa, "Microscopic origin of the Bekenstein-Hawking entropy," Physics Letters B, vol. 379, no. 14, pp. 99-104, 1996.

[54] C. Vafa, "Instantons on D-branes," Nuclear Physics B, vol. 463, no. 2-3, pp. 435-442, 1996.

[55] J. Maldacena, A. Strominger, and E. Witten, "Black hole entropy in M-theory," Journal of High Energy Physics, vol. 1997, no. 12, 1997.

[56] C. G. Callan, Jr. and J. M. Maldacena, "D-brane approach to black hole quantum mechanics," Nuclear Physics B, vol. 472, no. 3, pp. 591-608, 1996.

[57] G. T. Horowitz, J. M. Maldacena, and A. Strominger, "Nonextremal black hole microstates and $U$-duality," Physics Letters B, vol. 383, no. 2, pp. 151-159, 1996.

[58] G. T. Horowitz, D. A. Lowe, and J. M. Maldacena, "Statistical entropy of nonextremal four-dimensional black holes and $U$ duality," Physical Review Letters, vol. 77, no. 3, pp. 430-433, 1996.

[59] C. V. Johnson, R. R. Khuri, and R. C. Myers, "Entropy of $p \mathrm{D}$ extremal black holes," Physics Letters B, vol. 378, no. 1-4, pp. 7886, 1996.
[60] J. Maldacena, "The large $N$ limit of superconformal field theories and supergravity," Advances in Theoretical and Mathematical Physics, vol. 2, no. 2, pp. 231-252, 1998.

[61] D. Gaiotto, A. Strominger, and X. Yin, "Superconformal black hole quantum mechanics," Journal of High Energy Physics, no. 11, article 017, 2005.

[62] A. Castro, A. Maloney, and A. Strominger, "Hawking radiation by Kerr black holes and conformal symmetry," http://arxiv .org/abs/1004.0996.

[63] T. Hartman, K. Murata, T. Nishioka, and A. Strominger, "CFT duals for extreme black holes," Journal of High Energy Physics, no. 4, article 019, 2009.

[64] R. M. Wald, "Black hole entropy is the Noether charge," Physical Review D, vol. 48, Article ID R3427, 1993.

[65] T. Jacobson, G. Kang, and R. C. Myers, "Black hole entropy in higher curvature gravity," McGill/95-04; UMDGR-95-092, 1995.

[66] R. M. Wald, “The thermodynamics of black holes," Living Reviews in Relativity, vol. 4, no. 6, 2001.

[67] T. Jacobson and R. C. Myers, "Black hole entropy and highercurvature interactions," Physical Review Letters, vol. 70, no. 24, pp. 3684-3687, 1993.

[68] J. de Boer and M. Shigemori, "Exotic branes and nongeometric backgrounds," Physical Review Letters, vol. 104, no. 25, Article ID 251603, 2010.

[69] F. Denef and G. W. Moore, "How many black holes fit on the head of a pin?" General Relativity and Gravitation, vol. 39, no. 10, pp. 1539-1544, 2007.

[70] F. Denef and G. W. Moore, "Split states, entropy enigmas, holes and halos," http://arxiv.org/pdf/hep-th/0702146.pdf.

[71] I. Bena, C. W. Wang, and N. P. Warner, "Foaming three-charge black holes," Physical Review D, vol. 75, Article ID 124026, 2007.

[72] I. Bena, C.-W. Wang, and N. P. Warner, "Plumbing the abyss: black ring microstates," Journal of High Energy Physics, no. 7, article 019, 2008.

[73] V. Balasubramanian, V. Jejjala, and J. Simón, “The library of Babel: on the origin of gravitational thermodynamics," Journal of High Energy Physics, vol. 14, no. 12, pp. 2181-2186, 2005.

[74] V. Balasubramanian, M. Berkooz, A. Naqvi, and M. J. Strassler, "Giant gravitons in conformal field theory," Journal of High Energy Physics, no. 4, article 034, 2002.

[75] R. Emparan and H. S. Reall, “The end of black hole uniqueness," General Relativity and Gravitation, vol. 34, no. 12, pp. 20572062, 2002.

[76] J. L. Hovdebo and R. C. Myers, "Black rings, boosted strings, and Gregory-Laflamme instability," Physical Review D, vol. 73, no. 8, Article ID 084013, 2006.

[77] H. Elvang, R. Emparan, and A. Virmani, "Dynamics and stability of black rings," Journal of High Energy Physics, vol. 12, p. 74, 2006.

[78] R. Gregory and R. Laflamme, "Black strings and $\rho$-branes are unstable," Physical Review Letters, vol. 70, no. 19, pp. 2837-2840, 1993.

[79] G. Arcioni and E. Lozano-Tellechea, "Stability and critical phenomena of black holes and black rings," Physical Review D, vol. 72, no. 10, Article ID 104021, 2005.

[80] O. J. C. Dias, "Superradiant instability of large radius doubly spinning black rings," Physical Review D, vol. 73, Article ID 124035, 2006. 
[81] H. Lin, O. Lunin, and J. Maldacena, "Bubbling AdS space and 1/2 BPS geometries," Journal of High Energy Physics, no. 10, article 025, 2004.

[82] O. Lunin and S. D. Mathur, "AdS/CFT duality and the black hole information paradox," Nuclear Physics B, vol. 623, no. 1-2, pp. 342-394, 2002.

[83] O. Lunin and S. D. Mathur, "Statistical interpretation of the Bekenstein entropy for systems with a stretched horizon," Physical Review Letters, vol. 88, no. 21, Article ID 211303, 2002.

[84] S. D. Mathur, "Black hole size and phase space volumes," http://arxiv.org/abs/0706.3884.

[85] K. Skenderis and M. Taylor, "The fuzzball proposal for black holes," Physics Reports, vol. 467, no. 4-5, pp. 117-171, 2008. 

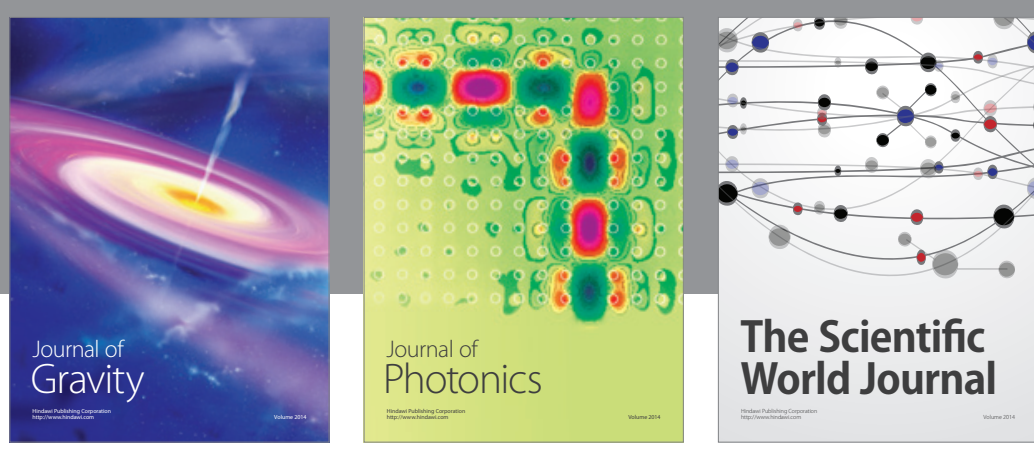

The Scientific World Journal
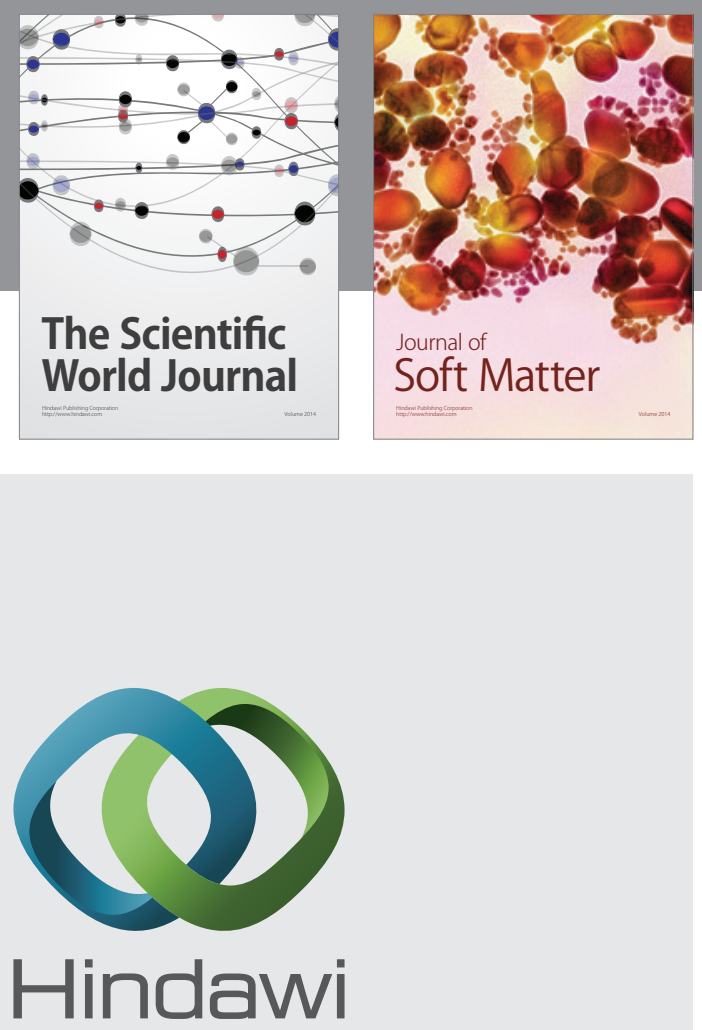

Submit your manuscripts at

http://www.hindawi.com

nternational Journal of

Statistical Mechanics
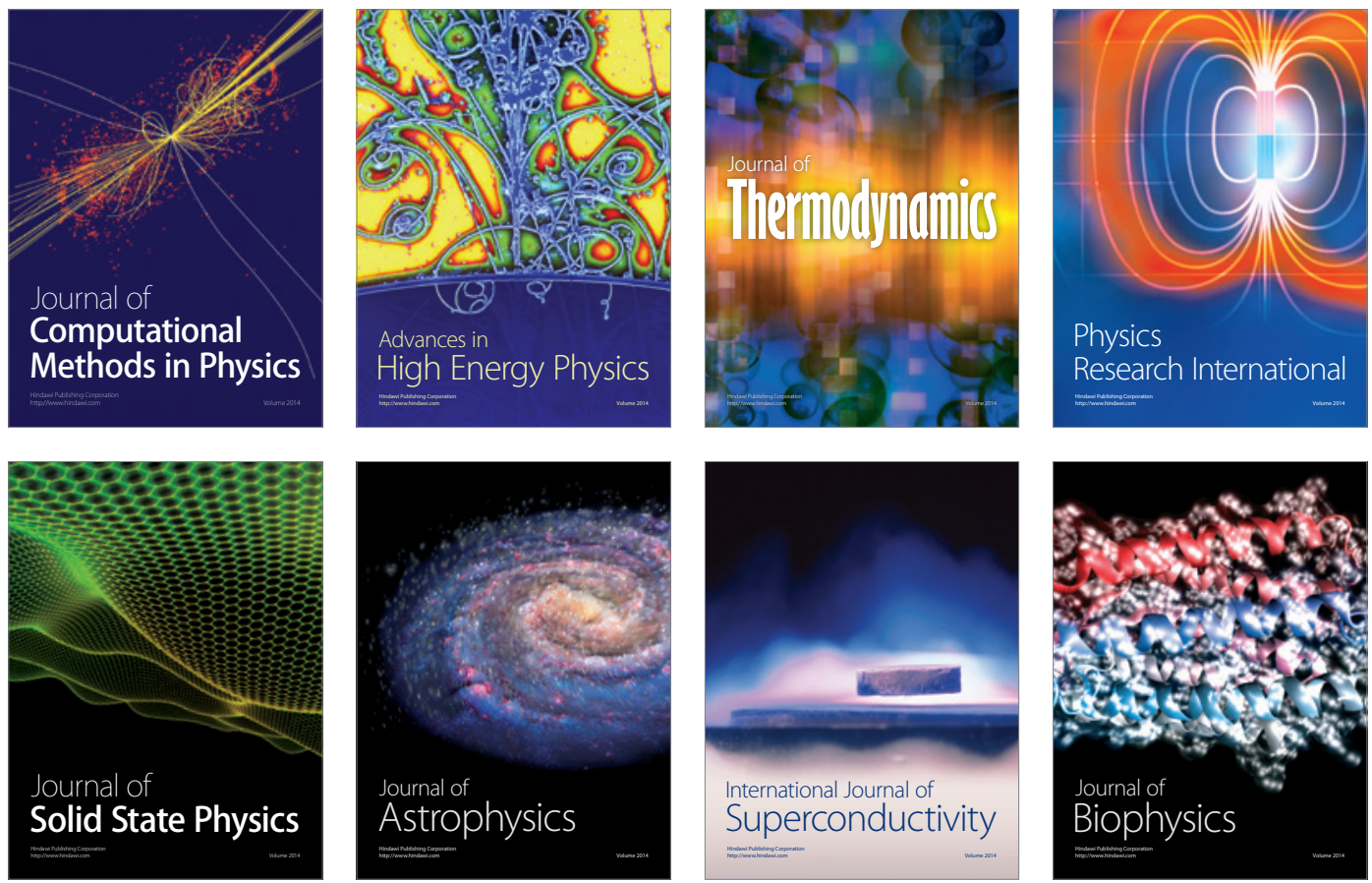
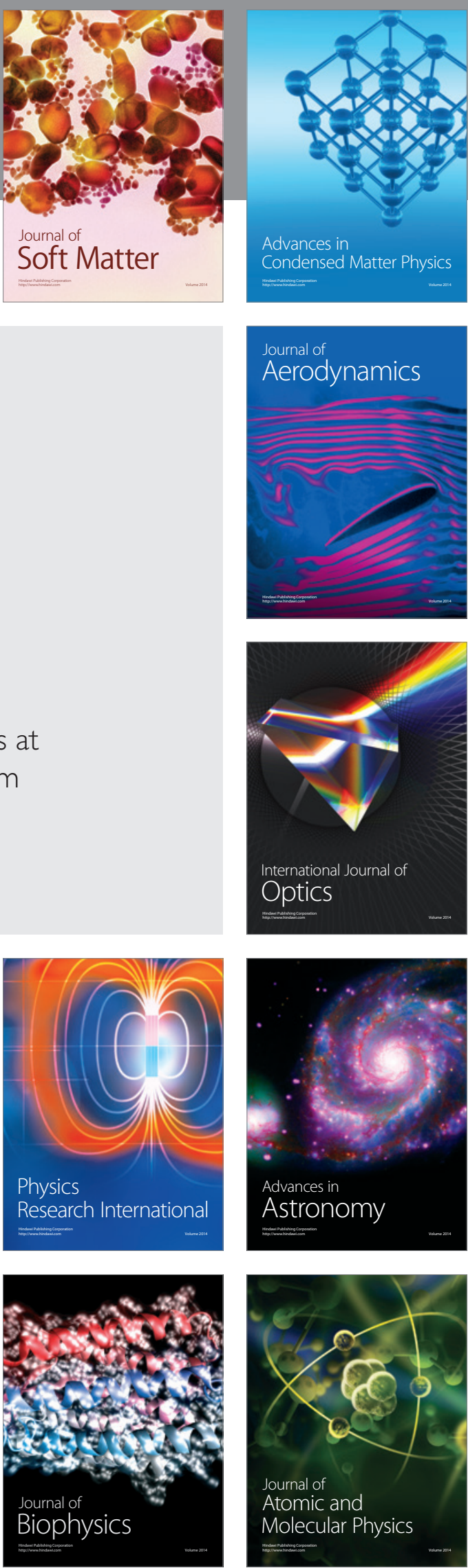